\title{
Las Ferias de Ciencia y Tecnología de Costa Rica y sus aportes a la educación secundaria
}

Science and Technology Fairs of Costa Rica and their contributions to Secondary Education

\author{
Volumen 18, Número 2 \\ Mayo-Agosto \\ pp. 1-43
}

Este número se publica el 1 de mayo de 2018

DOI: https://doi.org/10.15517/aie.v18i2.33170

\author{
Diego Armando Retana Alvarado \\ Bartolomé Vázquez Bernal \\ María Marta Camacho Álvarez
}

Revista indizada en REDALYC, $\underline{\text { SCIELO }}$

Revista distribuida en las bases de datos:

LATINDEX, DOAJ, REDIB, IRESIE, CLASE, DIALNET, SHERPA/ROMEO, QUALIS-CAPES, MIAR

Revista registrada en los directorios:

ULRICH'S, REDIE, RINACE, OEI, MAESTROTECA, PREAL, CLACSO 


\title{
Las Ferias de Ciencia y Tecnología de Costa Rica y sus aportes a la educación secundaria

\author{
Science and Technology Fairs of Costa Rica and their contributions to Secondary Education
}

\author{
Diego Armando Retana Alvarado ${ }^{1}$ \\ Bartolomé Vázquez Bernal² \\ María Marta Camacho Álvarez
}

\begin{abstract}
Resumen: En este artículo se analizan los aportes de las Ferias de Ciencia y Tecnología en la promoción de vocaciones científicas y tecnológicas en Educación Secundaria entre estudiantes cuyos proyectos de investigación resultaron seleccionados para participar en la Feria Nacional de Ciencia y Tecnología de Costa Rica de los años 2010 a 2013. Se identifican los componentes que motivan las decisiones de preferencia y elección de estudios superiores científico-tecnológicos en estudiantes de Bachillerato, así como la identificación de acciones del profesorado y aspectos por fortalecer para la efectiva promoción. Desde un diseño de triangulación concurrente mixto se encuestó a 45 estudiantes y 17 docentes costarricenses procedentes de colegios con modalidad académica, científica y técnica. Los resultados subrayan el desarrollo de competencias científicas, el fomento de actitudes favorables, el acercamiento al quehacer científico, los procesos de enseñanza y aprendizaje y el juzgamiento como componentes motivantes en la elección de carreras en esas áreas. Dicha promoción se reflejó en una alta preferencia por carreras tecnológicas y de corte científico en la escogencia al momento de ingresar a la universidad; motivada en parte, porque el profesorado de ciencias estimula a los estudiantes a realizar proyectos de investigación y brinda el acompañamiento durante su desarrollo y presentación en todas las etapas. Desde el Programa Nacional de Ferias de Ciencia y Tecnología se debe impulsar la formación permanente de los profesores en activo y la revisión del modelo pedagógico y organizativo vigente.
\end{abstract}

Palabras clave: investigación estudiantil, elección profesional, enseñanza secundaria, ferias de ciencia y tecnología.

\begin{abstract}
This article analyzes the contributions of Science and Technology Fairs in the promotion of scientific and technological vocations in Secondary Education among students whose research projects were selected to participate in the National Science and Technology Fair of Costa Rica in 2010 to 2013. It identifies the components that motivate the decisions of preference and choice of higher scientific-technological studies in high school students, as well as the identification of teachers' actions and aspects to be redefined for the effective promotion. From a mixed design of concurrent triangulation, 45 students and 17 teachers were surveyed. The results emphasize the development of scientific competencies, the promotion of favorable attitudes, the approach to the scientific work, the teaching and learning processes and the judging as motivating components in the choice of studies in those areas. Such influence was evident in a higher preference for technological careers and scientific studies at the time they first start university. Science teachers encourage students to carry out research projects and provide the accompaniment during its development and presentation in all stages. From the National Science and Technology Fairs Program, it is necessary the training of active teachers and the revision of the current pedagogical and organizational model.
\end{abstract}

Keywords: student research, occupational choice, secondary education, science and technology fairs.

\begin{abstract}
${ }_{1}$ Investigador en la Universidad de Huelva, España. Facultad Ciencias de la Educación. Departamento de Didácticas Integradas. Dirección electrónica: diegoarmando.retana@alu.uhu.es

${ }^{2}$ Docente e investigador en la Universidad de Huelva, España. Facultad Ciencias de la Educación. Departamento de Didácticas Integradas. Dirección electrónica: bartolome.vazquez@ddcc.uhu.es
\end{abstract}

3 Docente e investigadora en la Universidad de Costa Rica. Facultad de Educación. Escuela de Formación Docente. Sección de Educación Preescolar. mariamarta.camacho@gmail.com

Artículo recibido: 27 de noviembre, 2017

Enviado a corrección: 7 de febrero, 2018

Aprobado: 9 de abril, 2018 


\section{Introducción}

Actualmente, existe consenso en la Didáctica de las Ciencias Experimentales respecto a estimular las competencias científicas y competencias emocionales en los estudiantes, así que es clave para el desarrollo de la ciudadanía, desde una perspectiva de alfabetización científica que incluye la comprensión de la ciencia y sobre naturaleza de la ciencia (Lederman y Lederman, 2012). La alfabetización científica actúa como un puente que une los conocimientos científicos con la comprensión de la ciencia y sobre su naturaleza por parte del ciudadano común para que logre utilizar la ciencia y tecnología en su cotidianidad y en la toma de decisiones ante diversas cuestiones sociocientíficas que requieren diálogo, debate y argumentación. Por otra parte, el fomento de las vocaciones científicas y tecnológicas persigue el reclutamiento de científicos en diversas áreas, por tanto, los organismos gubernamentales y las universidades estatales deberían formular políticas para que esos procesos aseguren la igualdad de género.

En este contexto resulta oportuno el fomento de las vocaciones científicas y tecnológicas a través de la indagación como modelo didáctico alternativo (Rocard, Csermely, Jorde, Lenzen, Walberg-Henriksson, y Hemmo, 2007) y mediante otras actividades de promoción de la cultura científica como museos de ciencia, ferias científicas y tecnológicas, clubes de talento joven, campamentos, olimpiadas, semanas de la ciencia, entre otras que enlazan los sistemas educativos formales y la educación en ámbitos no formales e informales. En este artículo se analiza la contribución de las Ferias de Ciencia y Tecnología de Costa Rica en la promoción de las vocaciones científicas y tecnológicas en Educación Secundaria.

Las Ferias de Ciencia y Tecnología son actividades programadas donde los estudiantes plantean problemas, desarrollan proyectos de investigación en diversas temáticas científicas y aplicaciones, presentan y comunican sus hallazgos a las comunidades; representan un prisma que refleja factores cognitivos, afectivos y sociales en el aprendizaje escolar de las ciencias y sobre su naturaleza epistemológica, el desarrollo profesional del profesorado implicado y la cultura científica de las familias y los visitantes.

De acuerdo con Bencze y Bowen (2009), estas ferias tienen una larga tradición a nivel mundial, son útiles para promover la investigación por proyectos dentro y fuera del contexto escolar; pues facilitan el desarrollo de habilidades, concepciones sobre los fenómenos científicos y confianza para la resolución de problemas, influyendo no sólo en los conocimientos, sino autonomía, y pasan a contribuir en el proceso de enseñanza-aprendizaje 
y en la mejora de la comunidad donde viven (Araújo, 2015). En suma, son un mecanismo para el desarrollo de intereses científicos y motivación (Jones, 1991), aunque, como apunta (Finnerty, 2013), se necesita investigar más sobre la calidad de las experiencias de los estudiantes, incluyendo cantidad y tipo de instrucción antes y durante el proceso de la feria de ciencias.

En el contexto anglosajón, estas ferias tuvieron su génesis y popularidad durante el siglo XX; enmarcadas en el episodio histórico pre y post guerras mundiales, definieron la educación científica como fenómeno social y como estrategia de fomento de las funciones democráticas de la ciencia, aunque matizadas por intereses económicos y militares. Por ejemplo, la Feria Mundial de Nueva York (1939-1940) y la primera Feria Nacional de Ciencias de Philadelphia (1950) abrieron paso a la participación de escolares en la presentación de proyectos al público y jurado (Terzian, 2008; Terzian, 2013). Este hecho marcó el inicio de la Feria Internacional de Ciencia e Ingeniería (ISEF, por su acrónimo en inglés), un programa de la Sociedad para la Ciencia y el Público de los Estados Unidos de Norte América donde más de 3000 jóvenes preuniversitarios de aproximadamente sesenta países exponen sus proyectos de investigación científica e ingeniería y participan de un proceso de juzgamiento ante un conjunto de científicos incluidos receptores de Premios Nobel.

Estos antecedentes internacionales influenciaron otros contextos en Latinoamérica. Las Ferias de Ciencia y Tecnología se iniciaron en Costa Rica, en la década de los años sesenta, cuando se organizaron festivales de creatividad. A mediados de la década de 1970, la Facultad de Educación de la Universidad de Costa Rica promovió proyectos para fortalecer el interés de los estudiantes de la Cátedra de Enseñanza de las Ciencias. Además de incentivarles para la ejecución de un proyecto científico que les serviría como experiencia para, posteriormente, organizar una feria en la institución donde realizarían la Práctica Docente. Por ejemplo, de 1977 a 1983 la idea de la Feria se puso en práctica en el Liceo Laboratorio Emma Gamboa de la Universidad de Costa Rica, centro educativo que ha servido como "laboratorio de experimentación, innovación, e investigación pedagógica, para aplicar, evaluar y proyectar nuevas experiencias educativas” (García Fallas, Chaves Salas, Gurdián Fernández y Cedeño Suárez, 2002, p. 1).

A partir del 2014 se realizan una serie de propuestas de cambio de la Feria Nacional de Ciencia y Tecnología a solicitud de la dirección de la Escuela de Formación Docente, de la Comisión de Acción Asocial de esa unidad académica y de la Vicerrectoría de Acción 
Social de la Universidad de Costa Rica, sin embargo, a pesar de identificar en papel las estrategias y oportunidades de mejora, estas no se han llevado a cabo.

El Programa Nacional de Ferias de Ciencia y Tecnología (PRONAFECYT) es coordinado por el Ministerio de Ciencia, Tecnología y Telecomunicaciones, el Ministerio de Educación Pública, el Consejo Nacional para las Investigaciones Científicas y Tecnológicas y las universidades estatales (Universidad de Costa Rica, Universidad Nacional, Instituto Tecnológico de Costa Rica, Universidad Estatal a Distancia y Universidad Técnica Nacional), es responsable de la organización y el establecimiento de las políticas de las Ferias de Ciencia y Tecnología como enlace interinstitucional en el país. La Feria Nacional de Ciencia y Tecnología es una actividad que se realiza desde 1987 con la coordinación de la Universidad de Costa Rica. Este programa tiene como objetivo generar un cambio en la cultura científica mediante los procesos investigativos y la organización de las ferias institucionales, de circuitos escolares, regionales y la nacional.

Ese objetivo es similar al de otros programas iberoamericanos que promueven el gusto y la pasión por la investigación en los jóvenes, tales como Feria Nacional de Clubes de Ciencia (Uruguay), Feria Nacional Infantil y Juvenil de Ciencia, Tecnología e Innovación (Colombia), Feria de la Ciencia (Sevilla, España) y Exporecerca Jove (Barcelona). Estas ferias comparten similitudes, por ejemplo:

1) Son puntos de encuentro y comunicación entre jóvenes, docentes, investigadores y visitantes, donde se divulgan proyectos investigativos en diferentes áreas de la ciencia y tecnología.

2) Promueven en los estudiantes el desarrollo de competencias científicas y emocionales.

3) Los jóvenes investigadores elaboran una memoria de sus proyectos (informe escrito), proceden a su exposición y participan en un proceso de juzgamiento ante un Comité Científico de Revisión.

4) Las temáticas de los proyectos involucran aspectos del enfoque Ciencia, Tecnología y Sociedad. Por ejemplo, la Feria Nacional de Ciencia y Tecnología (FNCT) en varias ocasiones ha conmemorado las temáticas declaradas de interés internacional por la UNESCO; como el agua (XVII FNCT de 2013), la cristalografía (XVIII FNCT de 2014) y la luz (XIX FNCT de 2015).

Si bien estas actividades de alfabetización científica tan comunes a nivel internacional han marcado la ruta hacia una sociedad del conocimiento y la innovación, Bencze y Bowen 
(2009) sostienen que existen pocos hallazgos de investigación sobre estos fenómenos sociales. Algunos estudios sugieren que estas ferias motivan para trabajar en ciencia y revelan diferencias entre género, los hombres son más propensos a preparar proyectos de física, geología, matemática e informática en comparación con las mujeres (Arámbula Greenfield, 1995), quienes realizan proyectos en ciencias biológicas y sociales (Adamson, Foster, Roark y Reed, 1998).

Por ejemplo, en España, las exposiciones científicas escolares son de interés para motivar a los estudiantes y desarrollar otros factores asociados a la dimensión afectiva del aprendizaje de las ciencias (Oliva Martínez et al., 2004) y contribuyen en el desarrollo profesional de los profesores de Educación Secundaria, quienes intercambian experiencias con otros colegas y adquieren conocimientos didácticos al participar de procesos de formación continua y elaboración de módulos de exposición con sus estudiantes (Oliva Martínez, Matos Delgado y Acevedo Díaz, 2008).

Derivado de los planteamientos anteriores, partimos de la hipótesis que los estudiantes de Educación Secundaria al participar en las Ferias de Ciencia y Tecnología experimentan una práctica científica que les motiva en el desarrollo de su vocación científica y tecnológica que incide en las decisiones de preferencia y elección de carreras de ciencia y tecnología, en el momento de preparación para el ingreso a la universidad. Como señala Fouad (2007), la elección vocacional se construye a partir del conocimiento que cada persona tiene de sí misma y de la profesión, así como de la influencia del contexto social sobre el individuo.

Por tanto, consideramos que existen componentes de los procesos de Ferias de Ciencia y Tecnología que influyen en el desarrollo de la vocación y elección de carrera universitaria. En suma, creemos que el profesorado al motivar el desarrollo de proyectos de investigación se convierte en uno de esos pilares. Se espera que los resultados derivados de esta investigación permitan fortalecer los esfuerzos que se realizan desde las instituciones gubernamentales del PRONAFECYT para el impulso efectivo de las vocaciones en ciencia y tecnología y en la formación continuada de los profesores de todos los niveles.

Este estudio se desarrolló en el contexto natural de gestión, investigación y acción social de la Comisión Ejecutiva en el Programa Feria Nacional de Ciencia y Tecnología de la Universidad de Costa Rica. El estudio toma lugar en el seno de la controvertida aprobación y puesta en vigencia a nivel nacional en 2013 y 2014 respectivamente, de la "Reforma Integral al Decreto de Creación del Programa Nacional de Ferias de Ciencia y Tecnología e Inclusión en el Calendario Escolar", Decreto Ejecutivo N³7910 Ministerio de Educación Pública y 
Ministerio de Ciencia, Tecnología y Telecomunicaciones, en coherencia con las disposiciones generales del Plan Nacional de Ciencia, Tecnología e Innovación 2011-2014 (Ministerio de Ciencia y Tecnología, 2011) y coincidente con el periodo electoral costarricense de 2014.

Esa reforma ratifica la creación del PRONAFECYT, promueve la comprensión pública de la ciencia y tecnología y considera que la enseñanza de las ciencias representa una prioridad nacional para la promoción de vocaciones científicas y tecnológicas. Sin embargo, modifica las ferias de circuitos escolares en procesos de selección y evaluación de proyectos y limita la participación de los niños y las niñas del I Ciclo de la Educación Primaria en las etapas circuitales, regionales y la Feria Nacional de Ciencia y Tecnología, permitiéndoles compartir sus indagaciones únicamente en su centro educativo. El Decreto Ejecutivo $\mathrm{N}^{\circ}$ 37910 se derogó en 2015 porque no facilitaba una implementación pertinente al proceso de Ferias de Ciencia y Tecnología.

En este contexto institucional y organizativo se realizó el presente estudio con el propósito de analizar los aportes de las Ferias de Ciencia y Tecnología en la promoción de las vocaciones científicas y tecnológicas en Educación Secundaria. El objetivo general de la investigación se centró en distinguir los componentes del proceso que motivan las decisiones de preferencia y elección de estudios superiores científicos y tecnológicos, en estudiantes de Secundaria participantes en el proceso nacional en el periodo 2010-2013; así como en la identificación de las acciones que desarrolla el profesorado para motivar tales vocaciones y los aspectos por fortalecer para su efectiva promoción e incremento de su impacto social.

\section{Problemas de investigación}

Este estudio fue diseñado para obtener las respuestas a las siguientes preguntas: a) ¿Qué componentes de los procesos de Ferias de Ciencia y Tecnología motivan las decisiones de preferencia y elección de carreras científicas y tecnológicas en los estudiantes participantes de Educación Secundaria? b) ¿Qué acciones realiza el profesorado tutor en los procesos de enseñanza y aprendizaje de las ciencias para contribuir en la promoción de vocaciones científicas y tecnológicas? c) ¿Qué aspectos del proceso de Ferias de Ciencia y Tecnología deben fortalecerse para el impulso de una efectiva promoción de dichas vocaciones en Educación Secundaria? 


\section{Objetivos de investigación}

Esta investigación tiene como objetivo general analizar la contribución de las Ferias de Ciencia y Tecnología en las decisiones de preferencia y elección de carreras científicas y tecnológicas de la Educación Superior, por parte de los estudiantes de undécimo y duodécimo nivel, de instituciones educativas de las Direcciones Regionales de Educación San José Norte, San José Central, San José Oeste, San Carlos y Occidente, finalistas de las Ferias Nacionales de Ciencia y Tecnología durante el periodo 2010-2013.

De este objetivo se deriva la formulación de los siguientes más específicos:

a) Distinguir los componentes del proceso de Ferias de Ciencia y Tecnología que han motivado en los estudiantes participantes, la preferencia y elección de carreras científicas y tecnológicas.

b) Determinar las frecuencias de preferencia y elección académica de los estudiantes participantes, según el tipo de carrera universitaria y en función del género.

c) Identificar las acciones del profesorado de Educación Secundaria en los procesos de enseñanza y aprendizaje de las ciencias y del Programa Nacional de Ferias de Ciencia y Tecnología que han promocionado las vocaciones científicas y tecnológicas del estudiantado participante.

d) Distinguir los aspectos de las Ferias de Ciencia y Tecnología en Educación Secundaria, que deben fortalecerse para el impulso de una efectiva promoción de las vocaciones científicas y tecnológicas.

\section{Referente teórico}

\subsection{Modelo didáctico y organizativo de las Ferias de Ciencia y Tecnología en Costa Rica}

Las Ferias de Ciencia y Tecnología son actividades de alfabetización científica del ámbito formal que rescatan los procesos investigativos, estas sobresalen en la búsqueda de soluciones ante los problemas que sufren las comunidades y se enmarcan en el enfoque de Ciencia, Tecnología y Sociedad porque contribuyen en la cultura científica de la ciudadanía (Acevedo Díaz, 2004). Se preparan con el fin de atraer a niños, niñas y jóvenes pertenecientes al Sistema Educativo Nacional para que se vean estimulados a participar en la realización y presentación de proyectos de indagación o investigación escolar.

En estos procesos se expresan valores, emociones y actitudes hacia la ciencia; el desarrollo de habilidades en comunicación, destrezas interpersonales y sociales que 
contribuyen en el desarrollo de las competencias científicas. Asimismo, implican una mayor vinculación con el desarrollo de habilidades investigativas durante el desarrollo y presentación del proyecto (Rodríguez Morales y Ortiz Vargas, 2013).

En la Educación Primaria, las ferias promueven la reconstrucción del conocimiento, aspecto en el que es necesario un tratamiento interdisciplinario de los contenidos escolares. También, al ser un proceso que impacta en la enseñanza y el aprendizaje de las ciencias y otras áreas del quehacer humano, promueven el desarrollo integral del estudiantado al interactuar con otras personas de diferentes contextos. En la Educación Secundaria, los estudiantes realizan procesos de investigación de mayor complejidad en los que principalmente integran prácticas epistémicas con énfasis en ciencias básicas e ingeniería. Otros proyectos centran la mirada hacia los procesos de la investigación social.

En consonancia con las aportaciones de Cunningham y Kahle (2014), desde el ciclo de la investigación en las ciencias experimentales y la realización de proyectos científicos para presentar en las Ferias de Ciencia y Tecnología, los estudiantes del III Ciclo de la Educación General Básica y Educación Diversificada plantean preguntas y definen problemas para el desarrollo de teoría y testabilidad empírica; desarrollan y utilizan modelos, cuyo propósito es la explicación y predicción; planifican y realizan investigaciones, con el fin de probar hipótesis o evaluar el conocimiento; analizan e interpretan datos, atendiendo a cuestiones relacionadas con el mundo natural; utilizan el pensamiento matemático y computacional, a través de representaciones simbólicas y pruebas de hipótesis sobre la base de datos reales; construyen explicaciones y diseñan soluciones, con atención a la causalidad y pruebas empíricas para mejorar las explicaciones alternativas; argumentan desde las pruebas; obtienen, evalúan y comunican información.

Quienes desarrollan proyectos de desarrollo tecnológico enfatizan en la explicación de un problema práctico a través de la creación de un sistema o producto, analizan y evalúan modelos, adquieren datos para generar o evaluar nuevos diseños, atienden a multiplicidad de criterios en el análisis e interpretación de los datos, utilizan la matemática para diseñar prototipos a partir de la recolección o simulación de datos, seleccionan diseños a partir un conjunto de alternativas posibles, argumentan la solución propuesta con el proceso o producto tecnológico y finalmente comunican la información.

Los proyectos que evidencian construcción del conocimiento, innovación e impacto social pueden ser seleccionados en el juzgamiento para el otorgamiento de un reconocimiento y la participación en el Costa Rica Challenge. Ejemplos de estos proyectos 
de investigación estarían orientados a distinguir los factores sociales, afectivos y metacognitivos que inciden en el rendimiento académico y el aprendizaje científico de los estudiantes de un colegio de secundaria a través de la aplicación y análisis de un cuestionario o la construcción de un prototipo a través de elementos electrónicos que permita el tratamiento de las aguas residuales, la producción de biogás a partir de los desechos orgánicos de los comedores escolares, entre otros.

En consecuencia, las situaciones que promueven el aprendizaje exigen al estudiantado un esfuerzo de atención, comprensión y razonamiento para la resolución de problemas, así como su disposición para indagar en ambientes interactivos que le permiten un papel protagonista. En este sentido, el estudiantado que participa en estos procesos desarrolla una experiencia en la modalidad "aprendizaje por proyectos" (Crawford, 2014), en la que el profesorado se convierte en un facilitador y las preguntas fortalecen la investigación. La enseñanza no se limita a clases magistrales o centradas en modelos didácticos tradicionales; por el contrario, fomenta que el estudiantado se apropie del mejor laboratorio: su entorno. En otros casos, el proceso de investigación es mucho más diverso pues algunos estudiantes procedentes de colegios científicos o técnicos desarrollan su investigación en un laboratorio o taller. Entonces, los planteamientos anteriores se enmarcan en un modelo socioconstructivista o alternativo, en el que el estudiante, en interacción con sus pares, aprende a manipular los elementos del entorno y construyen argumentos a partir de las evidencias y el contraste de sus concepciones previas con las ideas científicas alternativas.

Estas ferias se realizan en las 27 Regiones Educativas del Ministerio de Educación Pública. Los procesos feriales en los niveles institucionales, de circuito escolar y de región educativa seleccionan, por medio de jueces, los mejores proyectos que luego participan en la Feria Nacional. Estos procesos fueron incorporados en el calendario escolar del Ministerio de Educación Pública mediante decreto MEP-MICIT 31900 y son obligatorios en todas las regiones educativas del país. Cabe destacar que antes de 2004 se calendarizaban algunas de las fechas importantes de los procesos y antes de ese año algunas regionales también realizaron ferias, por ejemplo, San José y San Carlos alrededor del 2000.

Las Ferias de Ciencia y Tecnología en Educación Secundaria se celebran mediante las siguientes categorías de participación: monografías, demostraciones de principios, procesos científicos y tecnológicos, proyectos de investigación científica y proyectos de desarrollo tecnológico, cada uno con características y con procedimientos que los distinguen. A partir del año 2006, se generó la categoría "Experiencias Científicas para Educación Preescolar" y, 
en 2017, se implementaron los lineamientos de las Experiencias Científicas para el I y II ciclo de la Educación Primaria denominada Quehacer científico y tecnológico. Los proyectos se ubican en nueve áreas temáticas: Biología; Ciencias Ambientales; Ciencias de la Computación; Ciencias de la Tierra y del Espacio; Ciencias Sociales y Humanidades; FísicaMatemática; Ingeniería y Tecnología; Química; Salud y Medicina (Camacho Álvarez, Durán Muñoz y Saborío Elizondo, 2007; Valencia Chacón, Camacho Álvarez, Monge Sandoval, Retana Alvarado y Martínez Sancho, 2010; Valencia Chacón et al., 2012; Valencia Chacón et al., 2018).

Los estudiantes realizan actividades que se enmarcan en un ciclo indagatorio comprendido por la selección y formulación del problema a través de preguntas de investigación, la definición de hipótesis (para aquellas categorías que lo requieran), la ejecución del plan acordado, el análisis, la puesta en común, la elaboración del informe y bitácora del proceso y la divulgación del proyecto.

Este modelo es coherente con la estructura de indagación propuesta por Bybee (2002), en la que los estudiantes se comprometen en la búsqueda de respuestas a preguntas científicamente orientadas, dando prioridad a la evidencia para responder a tales cuestiones, mediante un proceso de exploración de alternativas. Seguidamente, formulan explicaciones desde la evidencia y las conectan con el conocimiento científico para su evaluación. Posteriormente comunican y justifican sus explicaciones.

Los estudiantes registran y organizan sus ideas, observaciones, conocimientos, sentimientos y acciones en un cuaderno, registro electrónico u otro recurso al que se le denomina bitácora, ese documento representa la sistematización de todo el proceso investigativo seguido por los estudiantes y comprende desde sus inicios con la selección del tema hasta el desarrollo, la presentación y el juzgamiento del proyecto. La bitácora funciona como una estrategia metacognitiva porque facilita el aprendizaje de los conocimientos y procedimientos científicos mientras construyen una comprensión de la indagación y naturaleza de la ciencia en el transcurso de todas las etapas del proceso. Además, la presentación de la bitácora junto con el informe escrito y los formularios de inscripción representan el $60 \%$ de la evaluación en el juzgamiento.

Por otra parte, la exposición se sitúa dentro de un proceso de juzgamiento enfocado hacia la revisión del cumplimiento de las disposiciones establecidas para la presentación del proyecto, mediante el cual se verifican los logros adquiridos en función de los objetivos propuestos por los estudiantes en el trabajo elaborado. El Comité Científico de Revisión 
valora los trabajos escritos, asignando un 60\% en la evaluación; y posteriormente los jueces evalúan la presentación oral y las contribuciones en el área temática a la que corresponde el proyecto, otorgando un $40 \%$ en la valoración. Finalmente, aquellos proyectos de Educación Secundaria que evidencian un proceso de investigación riguroso, innovador y de impacto social pueden ser seleccionados para participar en el Costa Rica Challenge, donde por medio de juzgamiento se seleccionan los proyectos que representarán a Costa Rica en la Feria Internacional de Ciencia e Ingeniería que se celebra anualmente en diversas ciudades de los Estados Unidos.

\subsection{Elección de carrera universitaria, la vocación científica y sus componentes}

La elección de carrera es una decisión de parte del individuo, en la que integra sus visiones, significados y aspiraciones; igualmente, ajusta sus intereses y habilidades con determinado perfil profesional. En este sentido, los agentes educativos (maestros, padres, entre otros) que forman parte de la historia académica del estudiante, así como la relación de género y los estudios que asume la juventud, influyen en la elección profesional de esta (Montero, 2010). De acuerdo con Rodríguez Menéndez, Peña Calvo e Inda Caro (2016), la preferencia y el gusto personal por diversas opciones fundamentan sus decisiones académicas y predominan sobre otras razones como las salidas profesionales y el rendimiento académico. La elección vocacional de un estudiante considera también los recursos y el entorno en el que vive, sus expectativas económicas y el impacto social de la carrera, así como las limitaciones que su nivel económico le impone. En este sentido, factores como el nivel de ingresos que pueden obtener después de graduarse de una carrera universitaria y la demanda laboral de esa profesión para asegurarse un trabajo condicionan la elección de la vocación.

En este estudio entendemos la preferencia como la primacía que un estudiante posee sobre determinada carrera académica antes de realizar el proceso de admisión a la educación superior. Por otra parte, entendemos la elección como la escogencia de los estudios en el momento de realizar el ingreso a la universidad, facilitado u obstaculizado por factores intrínsecos o extrínsecos. Esta distinción es importante ya que tanto en el diseño metodológico como en el análisis de los resultados se parte de las decisiones de preferencia y de elección de carrera en el momento de la participación en los procesos de Ferias de Ciencia y Tecnología y el correspondiente al acceso a la educación superior 
respectivamente. Cabe aclarar que la escogencia de carrera también depende del proceso de selección que realiza la universidad para admitir estudiantes y este incluye el desempeño académico de los dos últimos años del bachillerato. En consecuencia, la profesión favorita con frecuencia no puede ser escogida, independientemente de la preferencia del estudiante.

Por otra parte, Vázquez Alonso y Manassero (2009a) mencionan que los estereotipos sociales provocan que las mujeres estudien profesiones catalogadas como femeninas (enfermería, educación, psicología y otras). En general, las profesiones científicas "femeninas" son un constructo social que suele ir asociado a la filosofía del "cuidado" o "servicio a los demás". Por su parte, los hombres eligen carreras de tipo científico y tecnológico basándose en valores como el éxito, la competición, el poder y otros; a diferencia de las mujeres que basan su elección a partir de motivos intrínsecos, es decir, se enfatiza en la satisfacción interior y valores personales.

Con respecto a la vocación científica y tecnológica, Vázquez Alonso y Manassero (2009b) a través de un análisis empírico demostraron que los factores afectivos y actitudinales influyen directamente en la vocación, la cual posee dos componentes: la elección de la carrera y las expectativas laborales. Estos autores afirman que las variables actitudinales de parte del estudiantado, así como la imagen de la ciencia, de las experiencias extraescolares, de la ciencia escolar y de las expectativas sobre el trabajo futuro tienen una alta capacidad predictiva de la vocación científica. Además, determinaron los predictores actitudinales más significativos: gusto por estudiar ciencias; la ciencia como un medio para generar mayores oportunidades de carrera y optar por excitantes trabajos; trabajar con máquinas o herramientas; inventar algo; y usar un equipo de ciencias.

Asimismo, Polino Carmelo (2012) destaca la importancia que tiene la escuela sobre la incidencia en las posibles elecciones de estudios futuros por parte de los jóvenes. Entre las actitudes favorables del estudiantado que se siente atraído hacia las profesiones científicas, destacan los hábitos para informarse sobre temas científicos y tecnológicos y un mayor aprecio por materias de ciencias que reciben en la escuela. No obstante, Vázquez Alonso y Manassero (2007) sostienen que la mayoría de los estudiantes que no eligen carreras de ciencia y tecnología se debe a que huyen del aburrimiento y de la dificultad que caracteriza a las clases de ciencias. Por lo tanto, consideran crucial la presencia de los aspectos afectivos, actitudinales y emocionales en la didáctica de las ciencias, pues afirman que las vocaciones pueden ser influidas significativamente por la orientación de la educación científica y los currículos escolares. 
Por otra parte, el planteamiento y la demostración de modelos teóricos sobre la percepción del alumnado hacia la indagación en ciencias (Vázquez Bernal y Jiménez Pérez, 2016) y su relación con otras variables como el género, el contexto socioeducativo y constructos como el autoconcepto y la autoeficacia se convierten en vertientes de investigación que, contextualizadas en un enfoque globalizador, podrían comprender y transformar la compleja realidad que caracteriza a estos procesos.

En este sentido, a través de la modelización de ecuaciones estructurales, se ha constatado que la autoeficacia es relevante por su relación con la capacidad de integración social en la comunidad científica y la predicción de continuar una carrera científica (Estrada, Woodcock, Hernández y Schultz, 2011) y que la motivación hacia las ciencias está altamente determinada por la autopercepción de autonomía y competencia (Lavigne, Vallerand y Miquelon, 2007) o por la importancia de la ciencia para sus futuras carreras (Glynn, Taasoobshirazi y Brickman, 2007). Además, utilizando este modelado estructural y a partir de los resultados de pruebas PISA, se encontró que los factores afectivos, como la actitud y motivación, contribuyeron al logro positivo en ciencias, en alumnado de 15 años de Turquía (Ozel, Caglak y Erdogan, 2013).

Las carreras científicas se entienden como profesiones que incluyen las ciencias básicas y de la salud en las que se realiza investigación sistemática para adquirir nuevos conocimientos, desarrollar teoría, explicar y predecir fenómenos, entre otras prácticas epistémicas. Las carreras tecnológicas son profesiones que utilizan los conocimientos generados por la ciencia para generar nuevos productos y procesos de utilidad para el ser humano y satisfacer sus demandas sociales, económicas y ambientales. La tabla 1 incluye la clasificación de las carreras universitarias empleadas en el marco de la presente investigación. 
Tabla 1

Categorización de carreras científicas, tecnológicas y sociales Ciencias Básicas Biología, Física, Meteorología, Geología, Matemática,

Educación Matemática, Ciencias Actuariales, Química, Gestión de los Recursos Naturales, Laboratorista

Carreras científicas

Ciencias de la Salud Químico.

\begin{tabular}{|c|c|c|}
\hline científicas & Ciencias de la Salud & $\begin{array}{c}\text { Enfermería, Medicina y Cirugía, Nutrición, Promoción de } \\
\text { la Salud, Odontología, Microbiología y Química Clínica, } \\
\text { Asistente de Laboratorio, Farmacia. }\end{array}$ \\
\hline \multirow[b]{2}{*}{$\begin{array}{l}\text { Carreras } \\
\text { tecnológicas }\end{array}$} & Tecnologías en Salud & $\begin{array}{c}\text { Imagenología Diagnóstica y Terapéutica, Terapia Física, } \\
\text { Salud Ambiental, Ortoprótesis y Ortopedia. }\end{array}$ \\
\hline & Ingenierías & $\begin{array}{c}\text { Ingeniería Civil, Ingeniería Eléctrica, Ingeniería } \\
\text { Industrial, Ingeniería Mecánica, Ingeniería Química, } \\
\text { Arquitectura, Ciencias de la Computación e Informática, } \\
\text { Computación con varios énfasis, Ingeniería Agrícola y } \\
\text { de Biosistemas, Salud Ambiental, Ingeniería en } \\
\text { Mecatrónica, Ingeniería Topográfica y Topografía, } \\
\text { Agronomía, Ingeniería de Alimentos, Ingeniería } \\
\text { Electromecánica Industrial, Ingeniería en Desarrollo } \\
\text { Sostenible, Marina Civil: Ingeniería Náutica y Transporte } \\
\text { Marítimo. }\end{array}$ \\
\hline \multirow[t]{3}{*}{$\begin{array}{l}\text { Carreras en } \\
\text { Ciencias } \\
\text { Sociales }\end{array}$} & Educación & $\begin{array}{l}\text { Educación Preescolar, Educación Primaria, Enseñanza } \\
\text { de las Ciencias Naturales, Enseñanza del Inglés, } \\
\text { Enseñanza del Francés, Enseñanza de la Filosofía, } \\
\text { Enseñanza de las Matemáticas, Enseñanza de los } \\
\text { Estudios Sociales y Educación Cívica, Enseñanza de la } \\
\text { Música, Enseñanza del Castellano y la Literatura, } \\
\text { Enseñanza de la Física, la Química y la Biología, } \\
\text { Enseñanza de la Psicología, Enseñanza de Educación } \\
\text { Física, Bibliotecología, Ciencias del Movimiento } \\
\text { Humano, Administración Educativa. }\end{array}$ \\
\hline & Ciencias Económicas & $\begin{array}{l}\text { Dirección de Empresas, Contaduría Pública, } \\
\text { Administración Pública, Administración Aduanera, } \\
\text { Comercio Exterior, Economía, Estadística. }\end{array}$ \\
\hline & Ciencias Sociales & $\begin{array}{l}\text { Ciencias de la Comunicación Colectiva (Publicidad, } \\
\text { Relaciones Públicas, Periodismo, Producción } \\
\text { Audiovisual y Multimedial, Comunicación Social, } \\
\text { Psicología, Ciencias Políiticas, Trabajo Social, Historia, } \\
\text { Archivística, Geografía, Antropología, Sociología. }\end{array}$ \\
\hline
\end{tabular}

Fuente: Elaboración propia a partir del listado de Carreras de Grado de la UCR, actualizado en junio de 2017.

El referente teórico y los antecedentes anteriores muestran la relevancia de la investigación complementada con los factores afectivos y actitudinales para el aprendizaje científico. Además, las Ferias de Ciencia y Tecnología de Costa Rica representan una oportunidad para los adolescentes porque les permite desarrollar su personalidad y perfilar su vocación científica y tecnológica. Estos antecedentes han sido importantes para el presente artículo, tanto desde la perspectiva teórica, como desde la metodológica. En consecuencia, han servido de referencia para delimitar el objeto de estudio y diseñar la metodología apropiada para su abordaje. 


\section{Metodología}

\subsection{Tipo de estudio}

El enfoque de esta investigación con alcance exploratorio y descriptivo recolecta, analiza y vincula datos cuantitativos y cualitativos para responder al problema. Así, el enfoque mixto proporciona una visión holística del fenómeno y permite la triangulación, complementariedad y credibilidad de los resultados (Hernández Sampieri, Fernández Collado y Baptista Lucio, 2010).

\subsection{Participantes}

En el estudio general se contó con la participación de 90 sujetos (45 estudiantes, 17 profesores, 17 asesores pedagógicos de Ciencias y 11 representantes de las universidades estatales y ministerios del gobierno nacional vinculados al PRONAFECYT). A continuación, presentamos las características de los participantes, los procedimientos de selección y datos derivados únicamente de los estudiantes y docentes, puesto que, sobre los demás se ha enfatizado en publicaciones previas (Retana Alvarado y Vázquez Bernal, 2016a; Retana Alvarado y Vázquez Bernal, 2016b).

El proceso de muestreo se realizó de la siguiente manera: En primer lugar, a través del sistema PROFERIAS y los formularios de inscripción del Comité Científico de Revisión Nacional se identificaron 62 estudiantes de bachillerato (undécimo y duodécimo año) participantes en la Feria Nacional de Ciencia y Tecnología en el periodo 2010-2013 procedentes de cinco regiones educativas de Costa Rica: San José Norte, San José Central, San José Oeste, San Carlos y Occidente.

En segundo lugar, se realizaron llamadas telefónicas y envío de correos electrónicos a esos estudiantes para informarles sobre el objetivo del estudio y solicitarles su participación. El correo electrónico incluía una invitación con enlace a la herramienta SurveyMonkey® donde completarían la encuesta. De las 62 invitaciones enviadas, 2 fueron devueltas y 15 no respondieron. Por tanto, se define a partir del criterio de accesibilidad y no por conveniencia una muestra constituida por 45 estudiantes (ver tabla 2). El 51\% de esos jóvenes son hombres y el $49 \%$ mujeres, con edades comprendidas entre los 16 a 18 años, con un nivel socioeconómico medio y bajo. Cuando estos estudiantes participaron en el estudio tenían entre 17 y 20 años. 
Tabla 2

Caracterización de la muestra de estudiantes participantes en el estudio por región y centro educativo en función del sexo, periodo 2010 a 2013

\begin{tabular}{ccccccccccc}
\hline & & $\mathbf{2 0 1 0}$ & $\mathbf{2 0 1 1}$ & $\mathbf{2 0 1 2}$ & $\mathbf{2 0 1 3}$ & $\mathbf{M}$ \\
\hline Región & Centro educativo & $\mathbf{H}$ & $\mathbf{M}$ & $\mathbf{H}$ & $\mathbf{M}$ & $\mathbf{H}$ & $\mathbf{M}$ & $\mathbf{H}$ & $\mathbf{M}$ & Total \\
\hline \multirow{4}{*}{ San José Norte } & Colegio Científico Costarricense San Pedro & 2 & 0 & 0 & 0 & 0 & 0 & 1 & 0 & 3 \\
& Liceo Laboratorio Emma Gamboa & 0 & 0 & 0 & 0 & 0 & 1 & 1 & 1 & 3 \\
& Colegio Saint Francis & 1 & 1 & 0 & 0 & 0 & 0 & 0 & 0 & 2 \\
& Colegio Técnico Profesional Don Bosco & 0 & 0 & 0 & 0 & 1 & 1 & 0 & 0 & 2 \\
& Colegio Superior de Señoritas & 0 & 0 & 0 & 0 & 0 & 0 & 0 & 1 & 1 \\
\hline San José Oeste & Liceo de Pavas & 0 & 0 & 0 & 0 & 0 & 1 & 0 & 0 & 1 \\
\hline & Colegio Científico Costarricense & 3 & 0 & 0 & 0 & 2 & 1 & 1 & 4 & 11 \\
& Colegio Técnico Profesional Nataniel Arias Murillo & 0 & 1 & 1 & 1 & 1 & 1 & 0 & 0 & 5 \\
San Carlos & Colegio Técnico Profesional Regional & 1 & 0 & 1 & 0 & 0 & 0 & 1 & 0 & 3 \\
& Colegio Técnico Profesional de Los Chiles & 0 & 0 & 0 & 0 & 2 & 0 & 0 & 0 & 2 \\
& Liceo Los Ángeles de Pital & 0 & 0 & 0 & 1 & 0 & 0 & 0 & 0 & 1 \\
\hline \multirow{3}{*}{ Occidente } & Colegio Científico Costarricense & 0 & 0 & 0 & 0 & 0 & 0 & 0 & 2 & 2 \\
& Colegio Técnico Profesional de Piedades Sur & 0 & 1 & 0 & 0 & 2 & 1 & 0 & 0 & 4 \\
& Liceo Experimental Bilingüe de Naranjo & 0 & 0 & 0 & 0 & 0 & 0 & 0 & 2 & 2 \\
& Colegio de Naranjo & 0 & 0 & 0 & 0 & 1 & 0 & 0 & 0 & 1 \\
& Colegio Nuestra Señora de Los Ángeles & 0 & 0 & 0 & 0 & 0 & 0 & 0 & 2 & 2 \\
\hline & Total & 7 & 3 & 2 & 2 & 9 & 6 & 4 & 12 & 45 \\
\hline
\end{tabular}

Nota: H: Hombres, M: Mujeres

Fuente: Elaboración propia a partir de la información recolectada, 2014.

El estudiantado participante provenía en su mayoría de colegios académicos pertenecientes al Sistema Nacional de Colegios Científicos de Costa Rica (16 estudiantes de las sedes San Pedro de Montes de Oca, San Carlos y San Ramón), así como colegios técnicos (16 estudiantes procedentes de 5 centros educativos); instituciones de Educación Secundaria en las que se promueve la ciencia y tecnología con mayor fuerza en el currículo oficial y que complementan su formación académica con el desarrollo de procesos investigativos para su participación en las Ferias de Ciencia y Tecnología.

En el caso particular de quienes estudiaron en Colegios Científicos Costarricenses, recibieron una formación académica enfocada principalmente en la adquisición de conocimientos y en el desarrollo de habilidades investigativas en Matemática, Física, Química, Biología e Informática. De forma paralela aplicaron los fundamentos científicos de cada una estas áreas en los laboratorios o en el campo para realizar sus proyectos de feria. Quienes estudiaron en colegios de modalidad científica realizaron el ingreso a través de rendimiento académico de excelencia en los niveles de sétimo, octavo y noveno, así como la aprobación del examen de admisión. De forma similar, el estudiantado proveniente de colegios técnicos ha recibido una formación especializada en áreas como Informática, Biotecnología, Electrónica, entre otras, lo cual le permite un acercamiento más vivencial al quehacer científico y tecnológico desde los procesos de enseñanza y aprendizaje. 
A su vez, se seleccionaron 17 docentes quienes fueron los tutores de estos estudiantes en los procesos de desarrollo y presentación de los proyectos de investigación en las cuatro etapas de las ferias. El profesorado tutor participó en varias ocasiones en la Feria Nacional de Ciencia y Tecnología en el periodo 2010-2013 e impartía las asignaturas de Física, Química, Biología, Matemática, Ciencias Sociales y Tecnologías como Electrónica y Biotecnología.

\subsection{Diseño de la investigación}

El presente estudio posee una naturaleza compleja y se aborda como un estudio expost-facto de naturaleza no experimental que incluye las percepciones de los estudiantes y sus tutores. Así, se aborda el objeto de estudio desde un diseño mixto, pues permite confirmar los resultados obtenidos tras la aplicación de las diferentes técnicas de recolección de datos. De esta forma, para la presente investigación se toma como referencia el diseño mixto de triangulación concurrente, propuesto por Hernández Sampieri et al. (2010). Se utiliza este diseño para validar de forma cruzada los datos cuantitativos y cualitativos, los cuales fueron recolectados simultáneamente. Para el presente estudio, este diseño abarca solamente la fase de recolección, análisis e interpretación.

\subsection{Instrumentos de investigación y técnicas de análisis de datos}

En el estudio general, la fase preliminar de recolección de datos inició con la aplicación de entrevistas semiestructuradas a 19 asesores procedentes de la Asesoría Nacional de Ciencias, Asesoría Nacional de Física y 17 Asesorías Regionales de Ciencias del Ministerio de Educación Pública procedentes de San José Central, San José Norte, San José Oeste, Pérez Zeledón, Los Santos, Occidente, San Carlos, Norte-Norte, Heredia (2 asesores), Sarapiquí, Turrialba, Puntarenas, Aguirre, Coto, Grande de Térraba y Limón, asimismo a 11 representantes del PRONAFECYT, cuyo propósito era obtener una aproximación a los componentes del proceso que contribuyen en las decisiones de preferencia y elección de carreras científicas y tecnológicas en Educación Secundaria (Retana Alvarado y Vázquez Bernal, 2016a; Retana Alvarado y Vázquez Bernal, 2016b).

Los asesores de Ciencias participaron en la etapa preliminar del estudio por su amplia experiencia en la docencia, formación continua de profesores en ejercicio y organización de las Ferias de Ciencia y Tecnología en sus regiones educativas, son quienes tienen contacto directo con los estudiantes, cuyos proyectos han sido seleccionados para participar en la 
Feria Nacional de Ciencia y Tecnología y por tanto, conocen las particularidades de su contexto (institución educativa, profesores, recursos económicos, entre otros).

La información se recopiló mediante grabación de voz y, posteriormente, se realizaron las transcripciones. A partir de la codificación de los datos cualitativos se identificaron las unidades de análisis relacionadas con el planteamiento del problema y se establecieron tres categorías, las cuales se fundamentan teóricamente a partir de las aportaciones de Retana Alvarado y Fallas Agüero (2013) y Vázquez Alonso y Manassero (2009b): "Factores afectivos y actitudinales", "Enseñanza y aprendizaje de las Ciencias" y "Procesos de desarrollo y presentación del proyecto".

Estas categorías concentran las ideas y temáticas similares identificadas por el investigador durante el análisis de la información. A partir de este sistema de categorías se diseñaron ad hoc dos cuestionarios que fueron validados a través del criterio experto de once especialistas nacionales e internacionales vinculados al PRONAFECYT (ver tabla 3). La validación se realizó mediante sesiones presenciales y virtuales con los expertos.

Tabla 3

Cargo y procedencia de los especialistas que participaron en la validación de los instrumentos de recolección de datos

\begin{tabular}{|c|c|c|}
\hline Código & Cargo & Procedencia \\
\hline$E-1$ & $\begin{array}{c}\text { Directora Feria Nacional de Ciencia y Tecnología } \\
\text { (2004-2007) } \\
\text { Directora Escuela de Formación Docente } \\
\text { (2008 a 2015) }\end{array}$ & Universidad de Costa Rica \\
\hline $\mathrm{E}-2$ & $\begin{array}{l}\text { Director Feria Nacional de Ciencia y Tecnología } \\
\qquad(2010-2013,2017-2018)\end{array}$ & Universidad de Costa Rica \\
\hline E-3 & $\begin{array}{c}\text { Director Feria Nacional de Ciencia y Tecnología } \\
\qquad(2014-2016)\end{array}$ & Universidad de Costa Rica \\
\hline$E-4$ & $\begin{array}{c}\text { Coordinadora Programa Nacional de Ferias de } \\
\text { Ciencia y Tecnología (2009-2018) }\end{array}$ & $\begin{array}{l}\text { Ministerio de Ciencia, } \\
\text { Tecnología y } \\
\text { Telecomunicaciones }\end{array}$ \\
\hline$E-5$ & $\begin{array}{c}\text { Asesor del Programa Nacional de Ferias de Ciencia } \\
\text { y Tecnología }\end{array}$ & $\begin{array}{l}\text { Universidad Nacional de Costa } \\
\text { Rica }\end{array}$ \\
\hline E-6 & Asesor de Ciencias Región Los Santos (2012-2018) & Ministerio de Educación Pública \\
\hline E-7 & Director Laboratorio Nacional de Nanotecnología & $\begin{array}{c}\text { Centro Nacional de Alta } \\
\text { Tecnología }\end{array}$ \\
\hline$E-8$ & Profesora e Investigadora Universitaria & $\begin{array}{l}\text { Universidad Central de } \\
\text { Venezuela }\end{array}$ \\
\hline
\end{tabular}

Fuente: Elaboración propia a partir de la información recolectada, 2014.

El primer cuestionario, de carácter mixto, con denominación C-1-E (ver anexo 1), estaba dirigido a los estudiantes y pretendía distinguir los componentes del proceso de Ferias de Ciencia y Tecnología que han motivado la preferencia y elección de carreras 
científicas y tecnológicas, así como determinar sus frecuencias según el tipo de carrera universitaria y en función del género.

El cuestionario está constituido por dos ítems abiertos y seis cerrados. En el primer y último ítem (ambos de carácter abierto) se recopilan datos referentes a los estudios superiores de preferencia y de elección en el momento de ingresar a la universidad, por lo que el participante anota la respuesta de acuerdo con su experiencia personal, esto sin presentársele la posibilidad de seleccionar entre un listado de opciones académicas. Cabe aclarar que no fue necesario acotar a los estudiantes el significado de "carreras científicas" y "carreras tecnológicas" dado que únicamente debían indicar el nombre de esas titulaciones. Posteriormente, para el tratamiento y análisis de los datos se tomó como referencia la categorización de las carreras universitarias que se indican en el marco teórico.

Los ítems 2, 3 y 4 del cuestionario C-1-E aportan información sobre el grado de acuerdo de los estudiantes respecto a la ciencia, las clases de Ciencias que recibieron cuando cursaron la Educación Secundaria y la participación en la Feria de Ciencia y Tecnología, a través de una escala tipo Likert, cuyos enunciados se presentan en forma de afirmaciones para medir la reacción del participante en cuatro niveles: nada de acuerdo (1), poco de acuerdo (2), bastante de acuerdo (3) y totalmente de acuerdo (4). Se incluyen cuatro alternativas dado que se perseguía que los sujetos brindaran una respuesta en la que estén a favor y así evitar el efecto del valor central. El ítem 5 indaga sobre la intensidad de algunas emociones experimentadas por el estudiantado en el desarrollo y presentación del proyecto. Finalmente, se incorporan en ítems de opción múltiple las expectativas de actividades profesionales (ítem 6) y los componentes del proceso de ferias que les motivó en la elección de ésta (ítem 7).

La aplicación del cuestionario C-1-E se realizó de manera virtual y en momentos distintos, pero en condiciones de aplicación similares, por medio de la utilización de la herramienta SurveyMonkey ${ }^{\circledR}$. Los estudiantes cumplimentaron el cuestionario de forma autoadministrada entre el 27 de enero y el 17 de marzo de 2014 (desde dos a cinco meses o hasta tres años después de su participación en la Feria Nacional de Ciencia y Tecnología), fueron informados acerca del propósito del estudio y la confidencialidad de los datos.

El segundo cuestionario mixto, con denominación C-2-T (ver anexo 2) está dirigido a los tutores y se constituye por un ítem cerrado y dos ítems abiertos. Este instrumento tenía como propósito distinguir los componentes motivantes en la preferencia y elección de estudios científicos y tecnológicos, identificar las acciones del profesorado que han 
promocionado las vocaciones científicas y tecnológicas y distinguir los aspectos del proceso a fortalecer para su efectiva promoción.

En el primero, el profesorado selecciona dentro de un conjunto de opciones, los componentes del proceso de Ferias de Ciencia y Tecnología, que considera fueron relevantes en las decisiones de preferencia y elección de la carrera científica y tecnológica de sus estudiantes. Los demás ítems son los siguientes: ¿Cuáles acciones ha desarrollado usted como docente y tutor en los procesos de Ferias de Ciencia y Tecnología para motivar la promoción y desarrollo de vocaciones científicas y tecnológicas en los jóvenes de undécimo y duodécimo nivel? ¿Cuáles aspectos considera usted que deben redefinirse para mejorar la promoción de éstas en las vocaciones científicas y tecnológicas y por tanto en la selección de carrera? El cuestionario C-2-T fue completado de manera autoadministrada por los profesores entre el 27 de enero y el 21 de abril de 2014.

Por su parte, la sistematización de los datos recolectados, de índole cuantitativa y cualitativa, se llevó a cabo mediante la herramienta SurveyMonkey ${ }^{\circledR}$, por medio de la utilización del sistema de archivador de datos, bajo el criterio de carpeta virtual por participantes encuestados (estudiantes y profesores); de tal forma que se pueden visualizar las respuestas individuales y grupales, así como un resumen tabulado y graficado con todos los datos de los participantes. La tabulación y el tratamiento de los datos cuantitativos se efectuó por medio de análisis estadístico descriptivo de frecuencias, al utilizar las herramientas SurveyMonkey ${ }^{\circledR}$ y una hoja de cálculo de Microsoft Office Excel con sus respectivas herramientas gráficas. Por último, se triangularon y compararon los datos con el diseño de triangulación concurrente mixto, para verificar la validez y confiabilidad de los resultados de la investigación; y se formularon las conclusiones generales desde el enfoque mixto.

\section{Resultados}

\subsection{Componentes motivantes en las decisiones de preferencia por una carrera científica y tecnológica desde la perspectiva de los estudiantes}

A continuación, se presentan los resultados de la aplicación del cuestionario C-1-E, cuyo propósito es distinguir los componentes del proceso que motivan la preferencia por estudios superiores científicos y tecnológicos. En primer lugar, los estudiantes expresaron su grado de acuerdo sobre la ciencia en la escala tipo Likert (ítem 2, ver figura 1). Para facilitar el análisis de los resultados expuestos en la figura 1, se presentan a continuación las cifras 
derivadas de la suma entre las frecuencias de valoración positiva (en donde el 4 corresponde a totalmente de acuerdo y el 3 a bastante de acuerdo) o negativa (en donde el 2 corresponde a poco de acuerdo y el 1 a nada de acuerdo) de la escala.

Figura 1. Porcentaje del estudiantado participante sobre la percepción de las ciencias según nivel de acuerdo, 2010-2013.

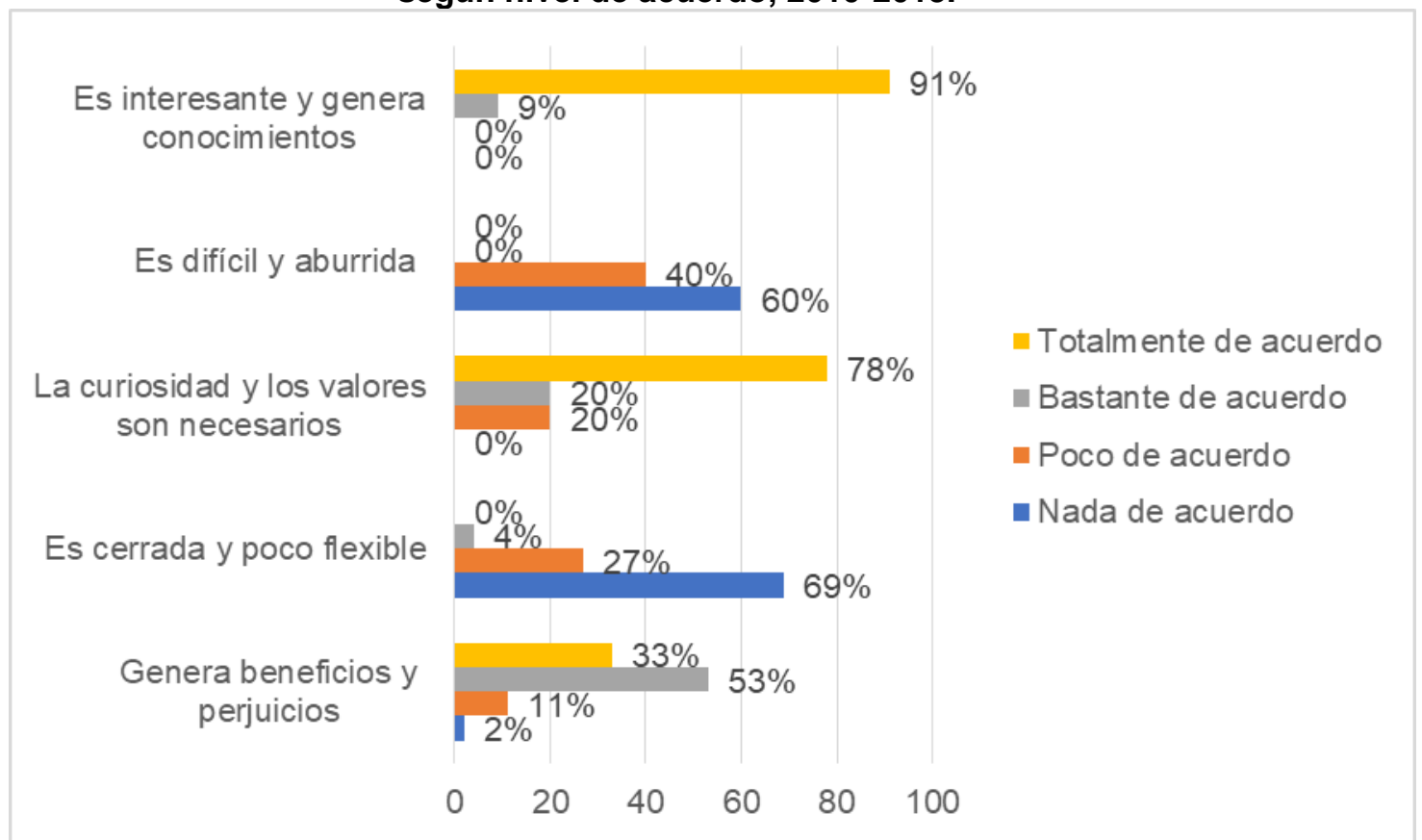

Fuente: Elaboración propia a partir de la información recolectada, 2014.

De acuerdo con los datos del gráfico, el 100\% del estudiantado opinó que está de acuerdo con que la ciencia es interesante y genera conocimientos para el progreso y la comprensión del mundo; el 98\% asegura que la curiosidad y los valores como la honestidad y la responsabilidad son necesarios en la ciencia; y el 86\% afirma que la ciencia genera beneficios y perjuicios. Además, el $96 \%$ de los participantes no están de acuerdo con que la ciencia es cerrada y poco flexible y el $100 \%$ no considera que la ciencia sea difícil y aburrida. La probabilidad de que la ciencia sea valorada como profesión aumenta en la medida en que el estudiantado tenga actitudes favorables sobre esta, por ejemplo, considera que la ciencia es atractiva, interesante, beneficiosa, fácil, abierta y flexible. Por tanto, los aspectos actitudinales positivos del estudiantado con respecto a la ciencia se convierten en un componente imprescindible en la vocación científica y tecnológica (Koballa y Glynn, 2007; Vázquez y Manassero, 2007). 
Por añadidura, los estudiantes expresaron su grado de acuerdo en la escala tipo Likert, con respecto a las clases de Ciencias que recibieron en su paso por la Educación Secundaria (ítem 3, figura 2). Para facilitar el análisis de los datos, se presentan a continuación las cifras resultantes de la suma entre las frecuencias de valoración positiva o negativa de la escala tipo Likert.

Figura 2. Porcentaje del estudiantado participante sobre la percepción de las clases de ciencias que recibieron en Educación Secundaria según el nivel de acuerdo, 2010-2013.

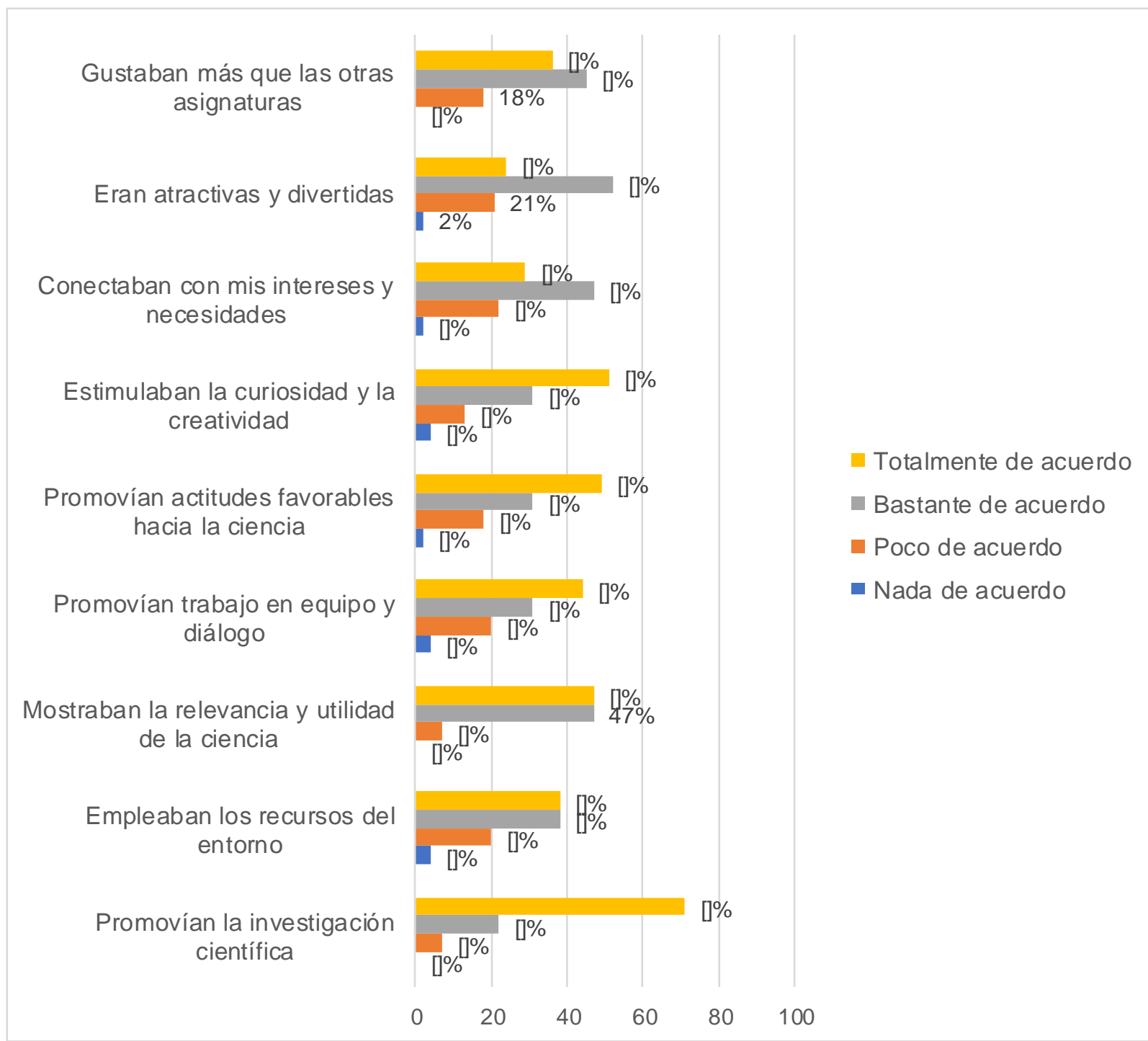

Fuente: Elaboración propia a partir de la información recolectada, 2014. 
Como se desprende de la figura 2, el 93\% del estudiantado está de acuerdo en que las clases de Ciencias recibidas promovían la investigación científica a través de la realización de un proyecto. También, el 76\% afirma estar de acuerdo en que se aprovechaban los recursos del entorno para la generación de conocimiento. De acuerdo con los datos, el 94\% de los participantes está muy de acuerdo en que las clases de ciencias mostraban la relevancia y utilidad de la ciencia para la vida y la sociedad. El 75\% afirma que las clases de ciencias promovían el trabajo en equipo y el diálogo, el 80\% en que impulsaban el desarrollo de actitudes favorables hacia la ciencia y el $82 \%$ que estimulaban la curiosidad y creatividad. Además, que el $76 \%$ del estudiantado afirma que las clases de ciencias conectaban con sus intereses y necesidades. De forma similar, el $76 \%$ asevera que estas lecciones eran atractivas y divertidas y el $81 \%$ opina estar muy de acuerdo con que las clases de ciencias le gustaban más que la mayoría de las otras asignaturas.

Como se desprende de la figura 2, el 93\% del estudiantado está de acuerdo en que las clases de ciencias recibidas promovían la investigación científica a través de la realización de un proyecto. También, el 76\% afirma estar de acuerdo en que se aprovechaban los recursos del entorno para la generación de conocimiento. De acuerdo con los datos, el 94\% de los participantes está muy de acuerdo en que mostraban la relevancia y la utilidad de la ciencia para la vida y la sociedad.

El $75 \%$ afirma que promovían el trabajo en equipo y el diálogo, el $80 \%$ en que impulsaban el desarrollo de actitudes favorables hacia la ciencia y el $82 \%$ que estimulaban la curiosidad y la creatividad. Además, que el $76 \%$ del estudiantado afirma que conectaban con sus intereses y necesidades. De forma similar, el $76 \%$ asevera que estas lecciones eran atractivas y divertidas y el $81 \%$ opina estar muy de acuerdo con que las clases de ciencias le gustaban más que la mayoría de las otras asignaturas.

La encuesta revela que los indicadores planteados con respecto a las clases de ciencias contribuyen en el desarrollo de la vocación científica y tecnológica y, por ende, en la posibilidad de elección de una carrera en estas áreas, por ejemplo, queda en evidencia que la mediación pedagógica promovía la investigación, era congruente con los intereses del estudiantado, fomentaba actitudes positivas hacia la ciencia y como asignatura gustaba más que las demás. El estudiantado encuestado valora positivamente a la ciencia, y valora también muy positivamente las clases recibidas durante su formación en educación media, en cuanto a diversos rasgos pertinentes y relevantes. Se puede considerar posible que lo 
positivo de estas clases haya incrementado la vocación científica y tecnológica de los estudiantes, ello ameritaría otro estudio.

Se observa, por ejemplo, que la ciencia y la tecnología, consideradas como posibilidades de estudio en el nivel universitario, son atractivas para casi el $90 \%$ de los estudiantes encuestados (Retana Alvarado y Vázquez Bernal, 2016a, Retana Alvarado y Vázquez Bernal, 2016b). Estos resultados son muy satisfactorios, pues es evidente la existencia de actitudes positivas hacia la ciencia y los procesos de enseñanza y aprendizaje, considerando la edad de los participantes, ya que como señalan Koballa y Glynn (2007), numerosos estudios reportan un decline de actitudes y emociones positivas de los estudiantes hacia las ciencias conforme progresan a través de los diferentes niveles educativos. De manera similar, los estudiantes expresaron su grado de acuerdo en la escala Likert, con respecto a su participación en las ferias (ítem 4, figura 3).

Figura 3. Porcentaje del estudiantado participante sobre la percepción en torno a su participación en las Ferias de Ciencia y Tecnología según el nivel de acuerdo, 2010-2013.

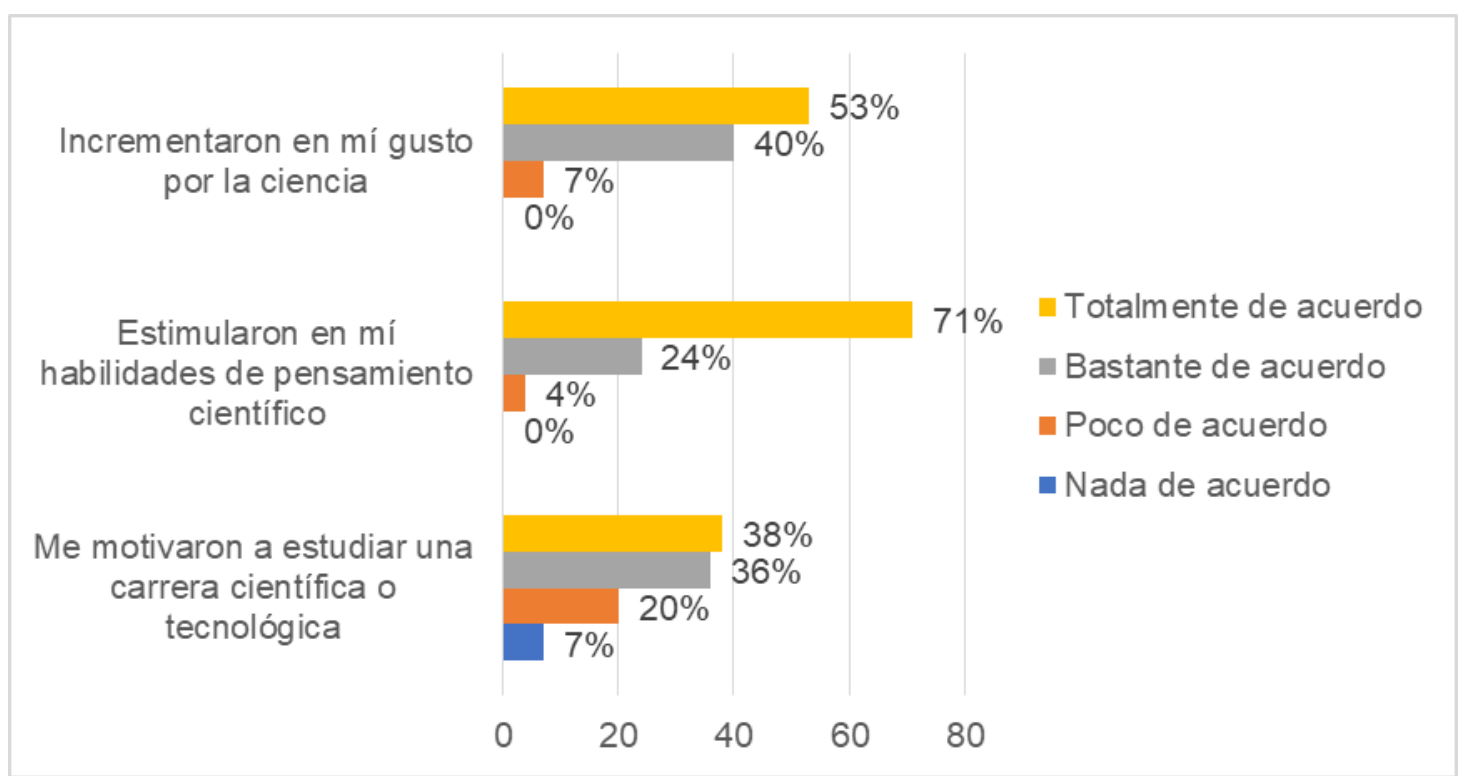

Fuente: Elaboración propia a partir de la información recolectada, 2014.

A continuación, se presentan las cifras resultantes de la suma entre las frecuencias de valoración positiva o negativa de la escala tipo Likert para facilitar el análisis de los datos. Según se evidencia en el gráfico anterior, el 95\% del estudiantado opinó que está muy de acuerdo en que su participación en las ferias le estimuló habilidades de pensamiento científico, tales como observar, medir, clasificar, comunicar, predecir, controlar variables, interpretar datos, formular hipótesis y experimentar. Los datos muestran, además, que el 
93\% de estos incrementó el gusto por la ciencia durante su participación en las ferias. De la misma manera, es evidente que su participación los motivó a estudiar una carrera científica o tecnológica, esto según el $74 \%$ de los estudiantes encuestados. Solamente el $26 \%$ opinó que no están de acuerdo con que las ferias los han motivado para realizar estudios universitarios vinculados con la ciencia y tecnología, esta frecuencia es muy alta y es un resultado inquietante por las particularidades de la muestra. En síntesis, la participación del estudiantado en dichas ferias le ha permitido incrementar sus habilidades y el gusto por la ciencia. En ese sentido, de cada cuatro estudiantes encuestados tres afirman haberse inspirado, por medio de estas ferias, para estudiar carreras científicas y tecnológicas.

En la línea del dominio afectivo, los estudiantes indicaron el nivel de intensidad de algunas emociones experimentadas mientras desarrollaron y presentaron el proyecto de investigación en la feria como respuesta al ítem 5 del instrumento C-1-E (ver figura 4).

Figura 4. Porcentaje del estudiantado participante sobre la percepción de las emociones experimentadas mientras desarrolla y presenta el proyecto en la Feria de Ciencia y Tecnología según el nivel de intensidad, 2010-2013.

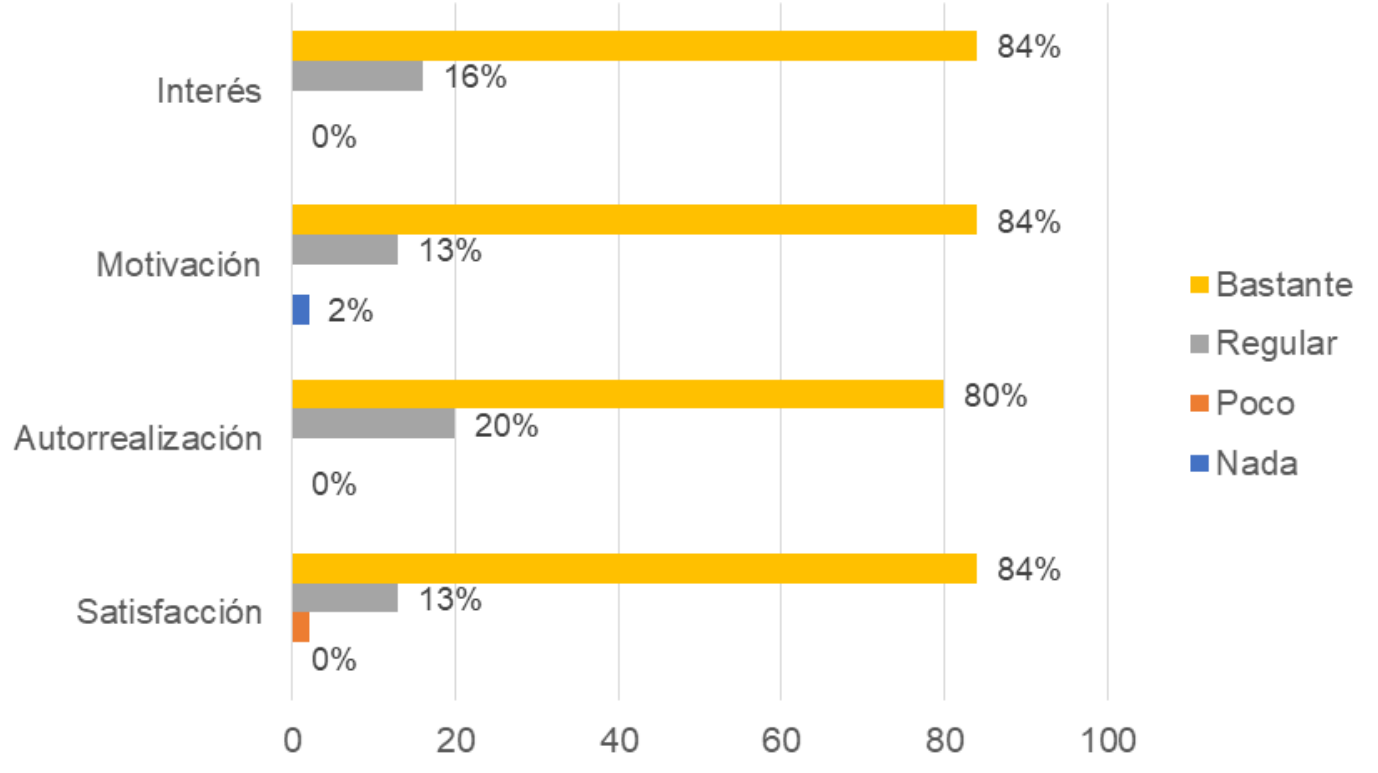

Fuente: Elaboración propia a partir de la información recolectada, 2014.

De acuerdo con los datos de la figura 4, el $84 \%$ del estudiantado sintió bastante satisfacción; el 80\% afirma haber sentido autorrealización; el 84\% experimentó motivación propia y/o por estímulo del docente, tutor o de la familia; y el 84\% restante aseveró mostrar interés mientras desarrolló y presentó el proyecto en la feria. Estos datos permiten reconocer la relevancia de las emociones por la fuerte influencia que ejercen en el desarrollo de la 
vocación científica y tecnológica. Al respecto, Mellado Jiménez et al. (2014), sostienen que las emociones están ligadas al aprendizaje de conocimientos concretos, por lo cual, desde el contexto de las ferias, los estudiantes se sienten motivados para seguir participando, pues les permite desarrollar conocimientos en la preparación, habilidades en la recopilación y sistematización de la información y, sobre todo, los tutores y profesores representan para ellos uno de los principales estímulos.

Ahora bien, como respuesta al ítem 6, los estudiantes señalaron dentro de un conjunto de posibles opciones, la o las actividades que deseaban realizar en mayor medida luego de participar en la feria. En primer lugar, el 87\% del estudiantado, después de su participación en estos procesos, desea realizar en mayor medida actividades como hacer, diseñar o inventar algo y, por ende, trabajar con máquinas y herramientas. En ese mismo sentido, el $29 \%$ desea construir y reparar objetos con las manos. Estos resultados están vinculados directamente con el $56 \%$ de la preferencia por carreras tecnológicas como las ingenierías, en las que se aplica el conocimiento científico para la generación de nuevos productos, procesos o servicios, y en las que se requiere la realización de actividades manuales. Es evidente entonces, la relación entre el tipo de carrera que los jóvenes deseaban estudiar, con el tipo de actividad que implica dicha profesión.

En segundo lugar, el $44 \%$ de los encuestados quiere ayudar a otras personas para mejorar su salud física y emocional. Estos resultados se relacionan con aproximadamente el $60 \%$ de la muestra que tenía interés por carreras científicas como Medicina, Microbiología y Farmacia. Finalmente, el 64\% afirma su deseo por trabajar en la protección del ambiente y dedicarse a la investigación sobre organismos vivos en el campo o en un laboratorio. Sin embargo, el grupo encuestado respondió con menor interés por carreras como Biología, Ingeniería en Biotecnología, Ingeniería en Agronomía e Ingeniería Ambiental (Retana Alvarado y Vázquez Bernal, 2016a, 2016b).

En términos generales, del ítem 7 se identifican componentes que contribuyen en la motivación por las decisiones de preferencia y elección de carreras científicas y tecnológicas (Retana Alvarado y Vázquez Bernal, 2016a, 2016b). Así, el 40\% del estudiantado opinó que el refuerzo de la autoestima durante el desarrollo y presentación del proyecto representó un componente que les motivó en la escogencia de una carrera científica o tecnológica. De igual forma, el $67 \%$ considera la motivación propia durante la elaboración del proyecto y el $49 \%$ la motivación que les brindó el profesorado en dicho momento, como aspectos influyentes en la escogencia. También, el $71 \%$ de los estudiantes aseveran que el aprovechamiento de las 
habilidades y destrezas para el desarrollo de competencias científicas como capacidad de análisis y síntesis, capacidad de explicar conceptos y disposición para indagar, así como un $53 \%$ representado por la aplicación de la teoría científica en la vida cotidiana forman parte de los componentes de los procesos de ferias que les motivaron en la escogencia de la carrera.

Además, les motivó la vivencia de procesos de exploración y experimentación, así como la visita a algún laboratorio o centro de investigación para la realización del proyecto, dichos componentes están representados por el $69 \%$ y el $27 \%$, respectivamente, de la opinión del estudiantado. Asimismo, la interacción con el público y con los jueces durante la exposición del proyecto y el área temática de la investigación, contribuyeron en la escogencia de carrera, esto de acuerdo con el $69 \%$ y el $27 \%$ del estudiantado participante. En otro orden de palabras, el 51\% le da importancia a la obtención de algún reconocimiento en cualquiera de las etapas de la feria; el 16\%, a su participación en la Feria Internacional de Ciencia e Ingeniería; y el 58\% del estudiantado considera que la feria es una inspiración para estudiar carreras científicas y tecnológicas y desempeñarse a futuro en trabajos relacionados con estas áreas. El 4\% del estudiantado afirmó que ninguno de los componentes de la feria, incluidos en el ítem 8, le motivó en la escogencia de una carrera universitaria.

Con referencia a lo anterior, aparecen anotados, por el estudiantado, otros componentes, tales como la satisfacción al obtener conocimientos que le permite crear nuevas invenciones para el beneficio de la sociedad. Además, afirma que mediante su participación en las ferias se incrementa la seguridad en sí mismos, lo cual les permite compartir sus descubrimientos con otras personas. Finalmente, a través de estos procesos de exploración e indagación, reafirman sus cualidades y descubren las diferentes opciones de carreras universitarias existentes.

\subsection{Componentes motivantes en las decisiones de preferencia por estudios superiores en ciencia y tecnología desde la perspectiva de los tutores}

En lo referente al conjunto tutor, como se deriva del ítem 1 del cuestionario C-2-T, el 94\% afirma que el contacto con la ciencia y la tecnología en la vivencia de procesos que permiten la exploración y la experimentación es un componente preponderante, el cual motivó a sus estudiantes en la escogencia de una carrera del área de la ciencia y tecnología. Asimismo, la opinión del conjunto tutor estuvo orientada, en su mayoría, al incremento del gusto, la afinidad y el interés por la ciencia (88\%). También, la motivación del profesorado hacia el estudiantado durante la elaboración del proyecto y el hecho de que la feria inspira el 
estudio de carreras científicas y tecnológicas; estuvieron representados cada uno por la opinión del $76 \%$ de estos encuestados.

El desarrollo de competencias científicas, al aprovechar las habilidades y destrezas del estudiantado; la motivación intrínseca del estudiante durante la realización de su proyecto; y la aplicación de la teoría científica en la vida cotidiana estuvieron representados por el 65\% (los dos primeros componentes) y por el 59\% (el último componente) de las percepciones del cuerpo tutor. Además, el refuerzo de la autoestima obtuvo el $59 \%$ de la opinión de estos, el área temática del proyecto el 53\% y la participación en la Feria Internacional de Ciencia e Ingeniería el 47\%. Igualmente, el conjunto tutor considera el reconocimiento, en alguna de las etapas de la feria; el proceso de juzgamiento y la interacción con el público, durante la exposición del proyecto; y la visita a un laboratorio o centro para la realización de la investigación, como aspectos influyentes en la escogencia de la carrera universitaria enfocada en ciencia y tecnología, estos están representados por el $41 \%$ para el primer componente y el $35 \%$ para los dos últimos.

Finalmente, dichos participantes consideran que al obtener la respuesta a alguna interrogante, a través de las metodologías investigativas, al estudiantado se le permite seguir indagando, lo cual promueve en estos la motivación, el incremento de la seguridad en sí mismos y el desarrollo de habilidades comunicativas, esto último al defender su proyecto de feria; estos componentes son insumos importantes para el desarrollo de la vocación científica y tecnológica y; por ende, para la elección de una carrera universitaria en estos ámbitos.

\subsection{Preferencia y elección de estudios superiores}

Los resultados globales derivados del ítem 1 y 8 del cuestionario C-1-E se han publicado en Retana Alvarado y Vázquez Bernal (2016a, 2016 b). En términos generales, antes de ingresar a la universidad, el $56 \%$ de los jóvenes participantes prefería estudios tecnológicos, seguido por los de corte científico, representados por el 33\%. El $11 \%$ de los adolescentes se orientaba por las Ciencias Sociales. Por ejemplo, en términos globales Medicina e Ingeniería en Electrónica son las carreras científicas y tecnológicas de mayor preferencia. Respecto a la elección, más de la mitad del estudiantado que llegó a ingresar lo hizo en la carrera de su preferencia y quienes ingresaron a la universidad lo hicieron a carreras tecnológicas, mayoritariamente, seguido por carreras científicas y de las Ciencias Sociales. 
En la tabla 4 se incluyen las frecuencias relativas para las carreras de preferencia y escogencia que los estudiantes indicaron en los ítems 1 y 8 , se han clasificado considerando la categorización de las carreras científicas, tecnológicas y sociales presentadas en el marco teórico. Estos resultados se basan en el sexo considerando un tamaño de muestra de 45 estudiantes (23 varones y 22 mujeres).

Antes de ingresar a la universidad, las mujeres tenían una mayor preferencia por carreras científicas en comparación con los hombres. No obstante, se aprecia una diferencia en puntos porcentuales de aproximadamente el $20 \%$ entre varones y mujeres respecto a carreras tecnológicas; es decir, los hombres prefieren mayoritariamente estudios relacionados con diversos tipos de Ingenierías a diferencia de las mujeres.

Tabla 4

Frecuencias relativas de preferencia y elección académica de los jóvenes participantes, por el tipo de carrera universitaria según sexo, 2010-2013. (valores relativos)

\begin{tabular}{ccccc}
\hline & \multicolumn{2}{c}{ Preferencia } & \multicolumn{2}{c}{ Escogencia } \\
\cline { 2 - 5 } Tipo de carrera & Hombres & Mujeres & Hombres & Mujeres \\
\hline Tecnológica & 65,2 & 45,5 & 52,17 & 31,82 \\
Científica & 26,1 & 36,3 & 21,74 & 9,10 \\
Ciencias Sociales & 8,70 & 18,2 & 8,69 & 13,63 \\
\hline No respondieron & 0 & 0 & 17,40 & 45,45 \\
\hline Total & 100 & 100 & 100 & 100 \\
\hline
\end{tabular}

Fuente: Elaboración propia a partir de la información recolectada, 2014.

Estos resultados son de gran relevancia porque se aprecia que el $81,8 \%$ de las mujeres prefiere carreras científicas y tecnológicas, solamente se diferencia en puntos porcentuales un 9,5\% respecto a los hombres, quienes alcanzan estos últimos el 91,3\% del interés en esas carreras. Los estudios en Ciencias Sociales poseen mayor preferencia de parte de las mujeres respecto a los hombres (una diferencia en puntos porcentuales equivalente al 9,5\%), lo cual está en consonancia con las aportaciones de Vázquez Alonso y Manassero Mas (2009a).

En relación con la escogencia de estudios superiores, a pesar de que las mujeres preferían las carreras científicas mayoritariamente que los hombres, solamente el $9,10 \%$ femenino inició estudios de esta índole. Por otra parte, la escogencia de carrera se focalizó mayoritariamente en estudios tecnológicos, tanto en hombres como mujeres, siendo los hombres quienes emprendieron más del $50 \%$ de estas carreras y las mujeres, aproximadamente la tercera parte de la escogencia en dichas titulaciones. Finalmente, la tendencia sobre la elección respecto a las Ciencias Sociales es similar al momento antes de 
ingresar a la universidad, pues las mujeres prefieren y eligen estos estudios mayoritariamente respecto a los hombres. Cabe decir, que el 17,4\% de los hombres y el $45,5 \%$ de las mujeres no se matricularon en la universidad, por razones fuera del alcance del presente estudio.

\subsection{Acciones del profesorado en las Ferias de Ciencia y Tecnología que han promocionado las vocaciones científicas y tecnológicas}

En este subapartado se presenta el análisis de los resultados obtenidos del ítem 2 del instrumento C-2-T que pretende identificar las acciones que realiza el profesorado tutor para promocionar dichas vocaciones en el estudiantado de Secundaria. De acuerdo con los datos, el cuerpo de docentes motiva a los discentes para que realicen investigación en temáticas de su interés con impacto social, al aprovechar el conocimiento científico adquirido en las clases de ciencias o el obtenido a través de la revisión de diferentes fuentes de información. Los impulsan para que refuercen su autoestima y reconozcan, tanto el valor de sus proyectos de investigación, como sus habilidades, esto con el fin de que descubran en qué ámbito del quehacer humano podrían desempeñarse. Por ejemplo, algunos docentes argumentan lo siguiente:

"El proceso motivador está relacionado con la confianza, asombro y gusto ante el trabajo propio. Mi acción fue enamorar a los estudiantes del proyecto, hacerles sentir que pueden marcar una diferencia con su trabajo y, ante todo, confiar en su capacidad e ingenio" (Profesor 8).

"Hacer que el estudiante se enamore del proyecto, para tener un mayor desempeño y eso les motiva a que hagan algo más y elegir una carrera. Eso les permite que encuentren su vocación" (Profesor 11).

El estudiantado vivencia procesos de enseñanza-aprendizaje en los que se le incita a encontrar la respuesta a diversas interrogantes, esto a través de retos por medio de los cuales adquieren conocimiento científico a partir de diversas estrategias de mediación, incrementando, así, el gusto por la ciencia y el desarrollo de actitudes favorables hacia la ciencia. Sobresale la aportación de la siguiente profesora quien orienta a sus estudiantes desde las clases de ciencias en el planteamiento, desarrollo y presentación del proyecto de feria: 
"Primero seleccionan el tema. Cuestionan el por qué lo seleccionaron y lo justifican de acuerdo con su realidad e interés. Van a los objetivos. Se les da un folleto con explicaciones básicas de formato APA para que aprendan a realizar referencias de libros, periódicos y referencias electrónicas. Se les ofrece suplementos de noticias científicas y tecnológicas. Se les incentiva a ir a la biblioteca y hacer entrevistas. Se les pide un anteproyecto en el que incluyen el tema, el marco teórico y la metodología. Se les revisa el trabajo y se les devuelve para que lo corrijan. Se insiste mucho en el formato de APA. Se realiza una preparación en el aula a través de la exposición y se refuerza las buenas exposiciones. Se realiza acompañamiento después de clase" (Profesora 16).

El profesorado se involucra al desempeñarse como cuerpo tutor de los proyectos de feria y brinda el acompañamiento necesario a lo largo de todo el proceso investigativo, apoyando al estudiantado con recursos académicos y económicos; guía a este por las diferentes etapas de la investigación, revisa sus avances y coordina con familiares y profesionales para la obtención de apoyo adicional.

\subsection{Aspectos por fortalecer para el impulso de una efectiva promoción de las vocaciones científicas y tecnológicas}

Como respuesta al ítem 3 del cuestionario C-2-T se distinguen los aspectos de los niveles de las Ferias de Ciencia y Tecnología que se deben fortalecer para la promoción de las vocaciones en ciencia y tecnología en los estudiantes participantes, desde la posición de los tutores. El profesorado plantea, por una parte, la necesidad de fortalecer su motivación, pues está desestimulado, con una cantidad reducida de identificación con las ferias, de conocimiento y experiencia en investigación; así como con ausencia de incentivo y exceso de labores propias del quehacer docente, las que le dificultan su participación comprometida. Por otra parte, el profesorado plantea la necesidad de motivación del estudiantado para que investigue y participe. Por ejemplo, una profesora argumenta lo siguiente:

"Por lo general, los estudiantes están motivados. He trabajado con estudiantes que tienen la convicción de querer trabajar en un proyecto para feria científica. Lo que se debería de modificar es la motivación con respecto a los docentes, he visto compañeros que trabajan estos procesos solo para cumplir un objetivo del plan de estudio, pero no se comprometen con grupos de estudiantes que están motivados. Puede ser que el tiempo que tienen dedicado en docencia es mucho y el incentivo para 
que lo realicen es mínimo, casi nulo, aparte que no tienen experiencia en desarrollar trabajos científicos por lo que se les dificulta ayudar al estudiante" (Profesora 2).

Aunado a lo anterior, el profesorado afirma que la mediación pedagógica debe incorporar dentro del currículo, la investigación, experimentación y participación de científicos que motiven a los discentes con su experiencia. Al profesorado, quien deberá tener una mejor preparación académica para impartir los fundamentos de la investigación, le corresponderá la promoción de procesos indagatorios estimulantes y sencillos que permitan la vinculación de la ciencia y tecnología con la cotidianidad. Al respecto, una docente expresa lo siguiente:

"El proceso de feria requiere planificación y mejora continua en el desarrollo de las propuestas de investigación. Con las modificaciones implementadas a finales de 2013 e inicios de 2014, se pierde de vista este horizonte y se aumenta el peso en la redacción científica, dejando de lado la experimentación por parte del investigador (proceso en el cual desarrollará sus capacidades y habilidades). La eliminación de ferias circuitales reduce la posibilidad de que los participantes mejoren sus propuestas de investigación y limita a discentes en formación de bajos recursos para que puedan acceder a la actividad, reduciendo el impacto formativo. No solo el estudiante que participa en la feria aprende, también el que la visita" (Profesora 3).

\section{Consideraciones finales}

Los componentes de las ferias que motivan la elección de carreras científicas y tecnológicas se enmarcan en las tres categorías de análisis a saber: "Factores afectivos y actitudinales", "Enseñanza y aprendizaje de las ciencias", "Desarrollo y presentación del proyecto".

Desde la categoría "Factores afectivos y actitudinales", los resultados evidencian que los jóvenes poseen actitudes favorables hacia la ciencia, por ejemplo, la consideran atractiva, interesante, beneficiosa, fácil, abierta y flexible. Reconocen que experimentaron emociones positivas como autorrealización, satisfacción, interés y motivación (intrínseca y extrínseca) durante el desarrollo y la presentación del proyecto.

Desde la categoría "Enseñanza y aprendizaje de las ciencias", la investigación revela que el profesorado que acompaña el proceso educativo de los estudiantes, la forma como se imparten las lecciones y la orientación científica con la que se desarrolla el currículo inciden, 
por un lado, en el desarrollo de la vocación científica y tecnológica del estudiantado y, por otra parte, en las decisiones de preferencia y elección de la carrera. Las clases de ciencias promueven la investigación científica a través de la realización de proyectos que surgen y se desarrollan en el contexto de aula, facilitan el aprovechamiento de los recursos del entorno para la generación de conocimiento, muestran la relevancia y la utilidad de la ciencia para la vida y la sociedad, promueven el trabajo en equipo y el diálogo, impulsan el desarrollo de actitudes favorables hacia la ciencia, estimulaban la curiosidad y la creatividad, así mismo establecen conexiones con los intereses y necesidades de los estudiantes. Además, ese ambiente de currículo reforzado, laboratorios, talleres y seguimiento o apoyo con profesores mejor formados favorece las vocaciones científicas y tecnológicas, especialmente en colegios científicos y técnicos, así que ese es el camino que debería seguirse en los demás colegios del país.

Los componentes de las Ferias de Ciencia y Tecnología de Costa Rica que motivan la preferencia y elección académico-profesional, guardan relación con factores influyentes en la elección de estudios superiores científico-tecnológicos por parte de jóvenes latinoamericanos. Estudios internacionales también identifican el interés por la ciencia, las clases desde una perspectiva práctica y los buenos docentes de ciencias (Vázquez Alonso y Manassero Mas, 2015), así como los hábitos para informarse sobre temas científicos y tecnológicos y un mayor aprecio por materias de ciencias que reciben en el centro educativo (Polino Carmelo, 2012). Otras investigaciones (Koballa y Glynn, 2007; Mellado Jiménez et al., 2014; Simpson, Koballa, Olive y Crawley, 1994; Vázquez Alonso y Manassero Mas, 2007; Zembylas, 2004) también evidencian la influencia que la dimensión afectiva de la enseñanza y el aprendizaje tiene sobre la elección de estudios superiores científico-tecnológicos.

Desde la categoría "Desarrollo y presentación del proyecto", que implica la forma en la que se realiza todo el proceso investigativo, los resultados muestran que las Ferias de Ciencia y Tecnología han permitido al estudiantado el desarrollo de competencias investigativas, ya que la vivencia de procesos de exploración y experimentación le permiten un contacto directo con la ciencia y tecnología. Además, la presentación del proyecto ante el público y la interacción con el conjunto de jueces durante el proceso de juzgamiento en las diferentes etapas de las ferias, le permiten al joven el desarrollo de habilidades comunicativas, así como el reconocimiento de la importancia del quehacer científico y tecnológico. 
Los aportes de las Ferias de Ciencia y Tecnología en la promoción de las vocaciones se reflejaron en una mayor preferencia por carreras tecnológicas y de corte científico, en la elección al momento de ingresar a la universidad; motivada en parte, porque el profesorado de ciencias estimula a los estudiantes a realizar proyectos de investigación. Por otro lado, acerca de las decisiones de preferencia y elección de carreras en esta investigación se concluye lo siguiente:

1) Las mujeres tienen una mayor preferencia por carreras científicas antes de ingresar a la universidad en comparación con los hombres.

2) Los hombres prefieren mayoritariamente los estudios tecnológicos relacionados con diversos tipos de Ingenierías que las mujeres.

3) La diferencia entre hombres y mujeres respecto a la totalidad de la preferencia de estudios superiores científico-tecnológicos es menor al 10\%.

4) Los estudios en Ciencias Sociales poseen una mayor preferencia de parte de las mujeres respecto a los hombres.

5) La elección de carrera se focalizó mayoritariamente en estudios tecnológicos, tanto en hombres como mujeres, siendo los hombres quienes emprendieron más de la mitad de estas carreras y las mujeres, aproximadamente la tercera parte de dicha elección, en el momento de matricularse en la universidad.

En relación con la escogencia de estudios superiores, a pesar de que las mujeres preferían las carreras científicas mayoritariamente que los hombres, solamente el 9,10\% femenino inició estudios de esta índole. Por otra parte, la escogencia de carrera se focalizó mayoritariamente en estudios tecnológicos, tanto en hombres como mujeres, siendo los hombres quienes emprendieron más del $50 \%$ de estas carreras y las mujeres, aproximadamente la tercera parte de la escogencia.

De acuerdo con Rossi Cordero y Barajas Frutos (2015), aún existe una asimetría en la representación de las mujeres con respecto a los hombres en los estudios científicos y tecnológicos debido a la persistencia de los estereotipos asociados a los estudios y ocupaciones en función del género. Sin embargo, se mantiene una diferencia de género en la efectiva entrada a estudios universitarios, siendo mucho mayor el porcentaje de mujeres que, a pesar de manifestar interés por el ingreso a carreras universitarias, finalmente no llega a matricularse. 
Por otra parte, los profesores afirman que realizan diversas acciones para motivar el desarrollo de las vocaciones en ciencia y tecnología. Los tutores indican que motivan a los estudiantes para que desarrollen y presenten proyectos en las ferias, suministrándoles el apoyo afectivo, académico y económico. Además, desde los procesos de enseñanza y aprendizaje orientan el proceso de indagación, gracias al desarrollo de actividades enfocadas en la selección y justificación del objeto de estudio, la búsqueda de información, la experimentación y las exposiciones en el aula. Finalmente, el profesorado brinda el acompañamiento durante su desarrollo y presentación en todas las etapas de la Feria de Ciencia y Tecnología.

También, los profesores explican la importancia de una revisión general del modelo de Ferias de Ciencia y Tecnología que se desarrolla en todo el país. Esa revisión debe partir de la integración de las instituciones que representan el PRONAFECYT, la calendarización de los procesos, la revisión de la obligatoriedad de participación, el presupuesto para el financiamiento de la actividad educativa, la participación de I Ciclo de la Educación Primaria, la relevancia de la ejecución de las ferias de circuitos escolares, las disposiciones generales de participación y los procesos de juzgamiento y reconocimiento. Esos procesos de revisión del modelo deben llevarse a cabo a través de sesiones de reflexión y desarrollo profesional con el profesorado, los asesores de ciencias y los miembros del PRONAFECYT. Por ejemplo, se deben organizar grupos focales y cursos de autoformación con modalidad presencial y virtual en todas las regiones educativas del país, de tal forma que se pueda estudiar y mejorar el impacto de las ferias en todos los niveles.

Por último, se concluye acerca de la necesidad de articular esfuerzos entre las instituciones académicas implicadas en el PRONAFECYT, en aras de alcanzar una promoción efectiva de las vocaciones científicas y tecnológicas de los jóvenes. De esta manera, un mayor estímulo al desarrollo profesional del profesorado se convierte en la herramienta para la mejora escolar y en la piedra angular para el éxito de las ferias. La formación docente es clave porque el profesor asume el rol de tutor y se topa con dificultades como la desmotivación, carencia de una formación científica investigadora suficiente, la desconfianza de que el estudiante sea capaz de realizar una investigación, su exceso de protagonismo como tutor y otras como la formulación de hipótesis, identificación de variables y diseño de experimentos (Ferrés Gurt, Marbà Tallada y Sanmartí Puig, 2015). Estas ferias implican una labor compleja y se requiere una participación comprometida de todos los agentes involucrados, comunicando una visión más cohesiva (Marquez, 2016). 


\section{Agradecimientos}

El estudio es financiado por la Universidad de Costa Rica. Cuenta con el aval de la Oficina de Asuntos Internacionales y Cooperación Externa, así como el aporte académico de la Escuela de Formación Docente de la Universidad de Costa Rica y el Departamento de Didácticas Integradas de la Universidad de Huelva (España).

\section{Referencias}

Acevedo Díaz, José Antonio. (2004). Reflexiones sobre las finalidades de la enseñanza de las ciencias: educación científica para la ciudadanía. Revista Eureka sobre Enseñanza y Divulgación de las Ciencias, 1(1), 3-16.

Adamson, Laura, Foster, Martha, Roark, Martha y Reed, Donna. (1998). Doing a science project: Gender differences during childhood. Journal of Research in Science Teaching, 35(8), 845-857.

Arámbula Greenfield, Teresa. (1995). An Exploration of Gender Participation Patterns in Science Competitions. Journal of Research in Science Teaching, 32(7), 735-748.

Araújo, Ana Vérica de. (2015). Feira de ciências: contribuições para a alfabetização científica na educação básica (Disertación doctoral). Universidad Federal do Ceará, Brasil. Recuperado de http://www.repositorio.ufc.br/handle/riufc/18922

Bencze, John Lawrence y Bowen, Gervase Michael. (2009). A national science fair: Exhibition support for the knowledge economy. International Journal of Science Education, 31(8), 2459-2483. Recuperado de https://www.tandfonline.com/doi/abs/10.1080/09500690802398127

Bybee, Rordger. (2002). Scientific Inquiry, Student Learning, and the Science Curriculum. En Rordger Bybee (Ed.), Learning science and the science of learning (pp. 25-35). Arlington, VA: NSTA Press.

Camacho Álvarez, María Marta, Durán Muñoz, Leticia y Saborío, Lidieth. (2007). Manual de Ferias de Ciencia y Tecnología: 2007-2009. San José, Costa Rica: Ministerio de Ciencia y Tecnología.

Crawford, Barbara. (2014). From Inquiry to Scientific Practices in the Science Classroom. En Norman Lederman y Sandra Abell (Eds.), Handbook of Research on Science Education (Vol. 2) (pp.515-599). New York: Routledge.

Cunningham, Christine y Kahle, Jane Butler. (2014). Precollege Engineering Education. En Norman Lederman y Sandra Abell (Eds.), Handbook of Research on Science Education (Vol. 2) (pp. 747-758). Nueva York: Routledge.

Estrada, Mica, Woodcock, Anna, Hernández, Paul y Schultz, Wesley. (2011). Toward a model of social influence that explains minority student integration into the scientific 
community. Journal of Educational Psychology, 103(1), 206-222. Recuperado de https://www.ncbi.nlm.nih.gov/pmc/articles/PMC3087606/

Ferrés Gurt, Concepció, Marbà Tallada, Anna y Sanmartí Puig, Neus. (2015). Trabajos de indagación de los alumnos: instrumentos de evaluación e identificación de dificultades. Revista Eureka sobre Enseñanza y Divulgación de las Ciencias, 12(1), 22-37. Recuperado de https://revistas.uca.es/index.php/eureka/article/view/2900/2584

Finnerty, Valerie. (2013). Can Participation in A School Science Fair Improve Middle School Students' Attitudes Toward Science and Interest in Science Careers? (Unpublished Doctoral Dissertation, University of Massachusetts Lowell). (UMI Number: 3570455)

Fouad, Nadya. (2007). Work and vocational psychology: Theory, Research, and application. Annual Review of Psychology, 58, 543-564. Recuperado de https://www.annualreviews.org/doi/abs/10.1146/annurev.psych.58.110405.085713

García Fallas, Jacqueline, Chaves Salas, Ana Lupita, Gurdián Fernández, Alicia y Cedeño Suárez, María Agustina. (2002). Los fundamentos curriculares del Liceo Laboratorio Emma Gamboa: construcción teórica a partir de una investigación participativa. Actualidades Investigativas en Educación, 2(2), 1-17. Recuperado de https://revistas.ucr.ac.cr/index.php/aie/article/view/8473

Glynn, Shawn, Taasoobshirazi, Gita y Brickman, Peggi. (2007). Nonscience majors learning science: A theoretical model of motivation. Journal of Research in Science Teaching, 44(8), 1088-1107. Recuperado de https://onlinelibrary.wiley.com/doi/abs/10.1002/tea.20181

Hernández Sampieri, Roberto, Fernández Collado, Carlos y Baptista Lucio, Pilar. (2010). Metodología de la Investigación (5ª ed.). México: McGraw-Hill.

Jones, Gail. (1991). Gender differences in science competitions. Science Education, 75(2), 159-167.

Recuperado

de https://onlinelibrary.wiley.com/doi/abs/10.1002/sce.3730750202

Koballa, Thomas y Glynn, Shawn. (2007). Attitudinal and Motivational Constructs in Science Learning. En Sandra Abell y Norman Lederman (Eds.), Handbook of Research on Science Education (pp.75-102). Nueva Jerseay: Lawrence Erlbaum Associates.

Lavigne, Geneviève, Vallerand, Robert y Miquelon, Paule. (2007). A motivational model of persistence in science education: A self-determination theory approach. European Journal of Psychology of Education, 22(3), 351-369. Recuperado de http://citeseerx.ist.psu.edu/viewdoc/download?doi=10.1.1.1003.3314\&rep=rep1\&type=p df

Lederman, Norman y Lederman, Judith. (2012). Nature of Scientific Knowledge and Scientific Inquiry: Building Instructional Capacity Through Professional Development. En Barry Fraser, Kenneth Tobin y Campbell McRobbie (Eds.), Second International Handbook of Science Education (Vol.1: pp. 335-359). Netherlands: Springer. 
Marquez, Fernando. (2016). The role of educational leadership on participation in the Costa Rican National Program of Science and Technology Fairs at Escuela Abraham Lincoln in the coastal region (Doctoral dissertation). University of Southern California, Estados Unidos.

Mellado Jiménez, Vicente, Borrachero Cortés, Ana Belén, Brígido Mero, María, Melo Niño, Lina Viviana, Dávila Acedo, María Antonia, Cañada Cañada, Florentina,... Bermejo García, María Luisa. (2014). Las emociones en la enseñanza de las ciencias. Enseñanza de las Ciencias, 32(3), 11-36. Recuperado de https://ddd.uab.cat/record/126039

Ministerio de Ciencia y Tecnología. (2011). Plan Nacional de Ciencia, Tecnología e Innovación 2011-2014. San José, Costa Rica: Ministerio de Ciencia y Tecnología.

Montero, María Teresa. (2010). Elección de carrera profesional: Visiones, promesas y desafíos. Ciudad Juárez, México: Universidad Autónoma de Ciudad Juárez.

Oliva Martínez, José María, Matos Delgado, Jesús y Acevedo Díaz, José Antonio. (2008). Contribución de las exposiciones científicas escolares al desarrollo profesional docente de los profesores participantes. Revista Electrónica de Enseñanza de las Ciencias, 7(1), 178-198. Recuperado de http://www.oei.es/historico/noticias/spip.php?article2036

Oliva Martínez, José María, Matos Delgado, Jesús, Bueno Garesse, Eduardo, Bonat Martínez, Manuel, Domínguez Ruiz, José, Vázquez Alonso, Ángel y Acevedo Díaz, José Antonio (2004). Las exposiciones científicas escolares y su contribución en el ámbito afectivo de los alumnos participantes. Enseñanza de las Ciencias, 22(3), 425440.

Ozel, Murat, Caglak, Serdar y Erdogan, Mehmet. (2013). Are affective factors a good predictor of science achievement? Examining the role of affective factors based on PISA 2006. Learning and Individual Differences, 24, 73-82. Recuperado de https://eric.ed.gov/?id=EJ1008338

Polino Carmelo, Andrés. (2012). Las ciencias en el aula y el interés por las carreras científico-tecnológicas: un análisis de las expectativas de los alumnos de nivel secundario en Iberoamérica. Revista Iberoamericana de Educación, 58, 167-191. Recuperado de https://dialnet.unirioja.es/servlet/articulo?codigo=4771062

Retana Alvarado, Diego Armando y Fallas Agüero, Manuel. (2013). Ferias de Ciencia y Tecnología en Costa Rica: una puerta a la innovación y al conocimiento. San José, Costa Rica: Sistema Editorial de Difusión Científica de la Investigación.

Retana Alvarado, Diego Armando y Vázquez Bernal, Bartolomé. (2016a). Influencia de las Ferias de Ciencia y Tecnología de Costa Rica en la elección de estudios superiores científicos y tecnológicos. En Jesús Sánchez Martín y Florentina Cañada Cañada (Eds.), Ciencias para comprender el mundo. Investigación e innovación en Didáctica de las Ciencias Experimentales (pp. 279-295). Madrid: Entinema. 
Retana Alvarado, Diego Armando y Vázquez Bernal, Bartolomé. (2016b). Ferias de Ciencia y Tecnología de Costa Rica: una experiencia que motiva la elección de carreras científicas y tecnológicas. Campo Abierto, 35(1), 13-30. Recuperado de https://dialnet.unirioja.es/ejemplar/448147

Rocard, Michel, Csermely, Peter, Jorde, Doris, Lenzen, Dieter, Walberg-Henriksson, Harriet y Hemmo, Valerie. (2007). Science Education Now: A Renewed Pedagogy for the Future of Europe. Bruselas: Comisión Europea.

Rodríguez Menéndez, María del Carmen, Peña Calvo, José Vicente e Inda Caro, María de las Mercedes. (2016). Esto es lo que me gusta y lo que voy a estudiar: Un estudio cualitativo sobre la toma de decisiones académicas en bachillerato. Revista Complutense de Educación, 27(3), 1351-1368.

Rodríguez Morales, Mayra y Ortiz Vargas, Rodolfo. (2013). La Feria de Ciencia y Tecnología desde la transversalidad: bases teóricas para la orientación en la gestión de proyectos ambientales en el bosque y la comunidad: antología 3, curso de capacitación a docentes en Ciencias, ciclo de conferencias del curso. San José, Costa Rica: Sistema Editorial de Difusión Científica de la Investigación.

Rossi Cordero, Andrea Estefanía y Barajas Frutos, Mario. (2015). Elección de estudios CTIM y desequilibrios de género. Enseñanza de las Ciencias, 33(3), 59-76. Recuperado de https://dialnet.unirioja.es/servlet/articulo?codigo $=5244405$

Simpson, Ronald, Koballa, Thomas, Oliver, Steve y Crawley, Frank. (1994). Research on the Affective Dimension of Science Learning. En Dorothy Gabel (Ed.), Handbook of Research on Science Teaching and Learning (pp. 211-234). New York: McMillan Publishing Company.

Terzian, Sevan. (2008). The 1939-1940 New York World's Fair and the Transformation of the American Science Extracurriculum. Science Education, 93, 892-914.

Terzian, Sevan. (2013). Science Education and Citizenship: Fairs, Clubs, and Talent Searches for American Youth, 1918-1958. Nueva York: Palgrave Macmillan.

Valencia Chacón, Nathalie, Arias Guido, Henry, Campos Quesada, Nelson, Martínez Sancho, Silas, Fernández Cordero, Lorena, Vega Baudrit, José Roberto...Umaña Campos, Annie (2018). Manual del Programa Nacional de Ferias de Ciencia y Tecnología de Costa Rica. San José, Costa Rica: PRONAFECYT-Ministerio de Ciencia, Tecnología y Telecomunicaciones.

Valencia Chacón, Nathalie, Camacho Álvarez, María Marta, Monge Sandoval, Johnnatan Andrés, Retana Alvarado, Diego Armando y Martínez Sancho, Silas. (2010). Manual de Ferias de Ciencia y Tecnología. San José, Costa Rica: Ministerio de Ciencia y Tecnología.

Valencia Chacón, Nathalie, De Lemos Morales, G., Camacho Álvarez, María Marta, Fernández Cordero, Lorena, Vega Baudrit, José Roberto, Muñoz Moya, Ana Cecilia, Rivera Rivera Keneth, Malavassi Rojas, Eduardo. (2012). Manual Ferias de Ciencia y Tecnología 2012-2014. San José, Costa Rica: Ministerio de Ciencia y Tecnología. 
Vázquez Alonso, Ángel y Manassero, María Antonia. (2007). En defensa de las actitudes y emociones en la educación científica (I). Revista Eureka sobre Enseñanza y Divulgación de las Ciencias, 4(2), 247-271. Recuperado de https://revistas.uca.es/index.php/eureka/article/view/3803/3376

Vázquez Alonso, Ángel y Manassero, María Antonia. (2009a). Patrones actitudinales de la vocación científica y tecnológica en chicas y chicos de secundaria. Revista Iberoamericana de Educación, 50, 1-15. Recuperado de https://rieoei.org/RIE/article/view/1879

Vázquez Alonso, Ángel y Manassero, María Antonia. (2009b). La vocación científica y tecnológica: predictores actitudinales significativos. Revista Eureka sobre Enseñanza y Divulgación de las Ciencias, 6(2), 213-231. Recuperado de http://venus.uca.es/eureka/revista/Volumen6/Numero 6 2/Vazquez Manassero 2009. pdf

Vázquez Alonso, Ángel y Manassero Mas, María Antonia. (2015). La elección de estudios superiores científico-técnicos: análisis de algunos factores determinantes en seis países. Revista Eureka sobre Enseñanza y Divulgación de las Ciencias, 12(2), 264277.

Vázquez Bernal, Bartolomé y Jiménez Pérez, Roque. (2016). Modelización de un constructo teórico sobre la percepción del alumnado en procesos de indagación en ciencias. Revista de Psicodidáctica, 21(1), 25-44. Recuperado de https://dialnet.unirioja.es/servlet/articulo?codigo $=5295483$

Zembylas, Michalinos. (2004). Emotion Metaphors and Emotional Labor in Science Teaching. Science Education, 88(3), 301-324. Recuperado de https://onlinelibrary.wiley.com/doi/abs/10.1002/sce.10116 


\section{Anexo 1.- Cuestionario C-1-E dirigido a estudiantes de Educación Secundaria participantes en la Feria Nacional de Ciencia y Tecnología en el periodo 2010-2013}

1. Indique la carrera universitaria que más le interesaba en su último año de Bachillerato

2. De las siguientes ideas que aparecen a continuación, señale su grado de acuerdo sobre la ciencia.

\begin{tabular}{|c|c|c|c|c|}
\hline \multirow{2}{*}{$\begin{array}{l}\text { La ciencia es interesante y genera conocimientos para el progreso y la } \\
\text { comprensión del mundo. }\end{array}$} & 1 & 2 & 3 & 4 \\
\hline & & & & \\
\hline La ciencia es difícil y aburrida & & & & \\
\hline La curiosidad y los valores son necesarios en la ciencia. & & & & \\
\hline La ciencia es cerrada y poco flexible & & & & \\
\hline La ciencia generara beneficios y perjuicios. & & & & \\
\hline
\end{tabular}

1: Nada de acuerdo, 2: Poco de acuerdo, 3: Bastante de acuerdo, 4: Totalmente de acuerdo.

3. De las siguientes ideas que aparecen a continuación, señale su grado de acuerdo sobre las clases de ciencias que usted recibía cuando estaba en Educación Secundaria.

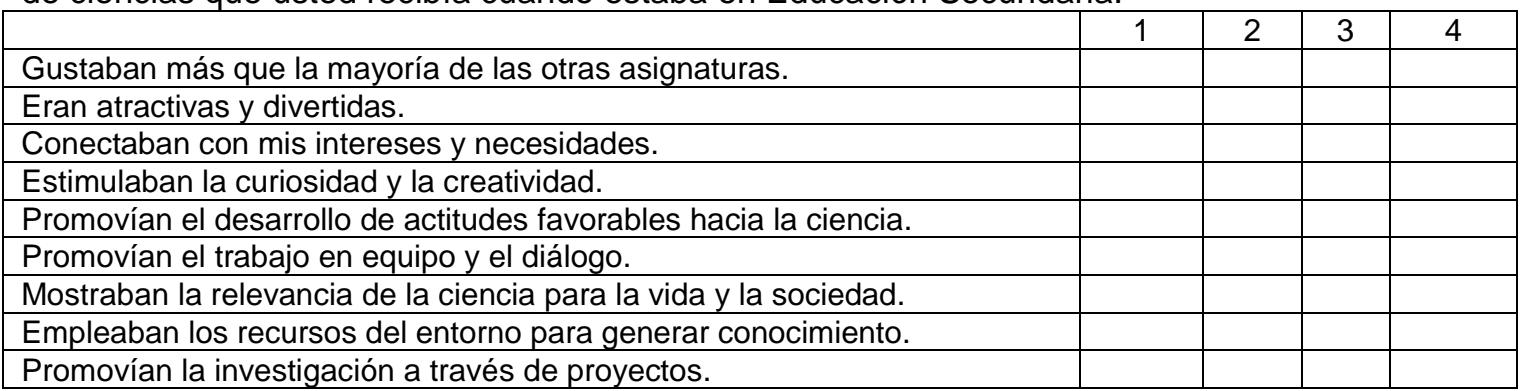

1: Nada de acuerdo, 2: Poco de acuerdo, 3: Bastante de acuerdo, 4: Totalmente de acuerdo.

4. De las siguientes ideas que aparecen a continuación, señale su grado de acuerdo sobre su participación en las Ferias de Ciencia y Tecnología.

Incrementaron en mí el gusto por la ciencia.

Estimularon en mí habilidades de pensamiento científico asociadas a procesos de hacer, pensar y comunicar.

Me motivaron a estudiar una carrera científica o tecnológica.

1: Nada de acuerdo, 2: Poco de acuerdo, 3: Bastante de acuerdo, 4: Totalmente de acuerdo.

5. Indique con cuánta intensidad sintió usted las siguientes emociones mientras desarrolló y presentó el proyecto en la Feria de Ciencia y Tecnología.

\begin{tabular}{|c|c|c|c|c|}
\hline & 1 & 2 & 3 & 4 \\
\hline Interés & & & & \\
\hline Motivación (propia y/o por estímulo del docente, tutor(a) o de la familia) & & & & \\
\hline Autorrealización & & & & \\
\hline Satisfacción & & & & \\
\hline
\end{tabular}

1: Nada, 2: Poco, 3: Regular, 4: Bastante.

6. De las siguientes actividades, ¿cuál deseaba realizar en mayor medida luego de participar en la Feria de Ciencia y Tecnología?

$\Gamma \quad$ Ayudar a otras personas para mejorar su salud física y emocional.

$\Gamma \quad$ Investigar sobre organismos vivos en el campo o en un laboratorio.

$\Gamma \quad$ Trabajar en la protección del ambiente.

$\sqsubset$ Construir o reparar objetos con las manos.

$\square$ Hacer, diseñar o inventar algo. 
$\Gamma \quad$ Trabajar con máquinas o herramientas.

$\Gamma \quad$ Ninguna de las anteriores.

$\Gamma$ Otro (especifique)

7. Seleccione cuál o cuáles de los siguientes componentes del proceso de Ferias de Ciencia y Tecnología considera usted que le motivaron en la escogencia de la carrera.

$\Gamma \quad$ Gusto, afinidad e interés por la ciencia.

$\Gamma$ Refuerzo de la autoestima en el desarrollo y presentación del proyecto

$\Gamma$ Aprovechamiento de habilidades y destrezas para el desarrollo de competencias científicas.

Г Aplicación de la teoría científica en la práctica.

$\Gamma$ El contacto con la ciencia y la tecnología al vivenciar procesos que permiten la exploración y la experimentación.

Г Motivación propia durante la elaboración del proyecto.

Г Motivación por parte del docente durante la elaboración del proyecto.

$\Gamma$ Visita a algún laboratorio o centro de investigación para realizar el proyecto de feria.

$\Gamma \quad$ Área temática del proyecto.

Г La interacción con el público y con las y los jueces durante la exposición del proyecto.

$\Gamma$ Obtención de algún reconocimiento en alguna de las etapas de la feria.

Г Participación en la Feria Internacional de Ciencia e Ingeniería u otras.

$\Gamma \quad$ La feria inspira para estudiar carreras científicas y tecnológicas y desempeñarse a futuro en trabajos relacionados con esas áreas.

Г Ninguno de los anteriores.

Г Otro (especifique)

8. Si se encuentra estudiando en una universidad, indique el nombre de la carrera en la que está matriculado. Si no está matriculado ha finalizado la encuesta. 


\section{Anexo 2.- Cuestionario C-2-T dirigido a tutores de los estudiantes de Educación Secundaria finalistas en la Feria Nacional de Ciencia y Tecnología en el periodo 2010- 2013.}

1. Seleccione cuál o cuáles de los siguientes componentes del proceso de Ferias de Ciencia y Tecnología considera usted que promueve la escogencia de una carrera científica o tecnológica en las y los estudiantes de undécimo y duodécimo año.

Г Al participar en la Feria de Ciencia y Tecnología incrementa el gusto, la afinidad y el interés por la ciencia.

$\square$ Refuerzo de la autoestima en el desarrollo y presentación del proyecto.

$\Gamma \quad$ Aprovechamiento de las habilidades y destrezas para el desarrollo de competencias científicas.

$\square$ Aplicación de la teoría científica en la práctica.

$\square$ El contacto con la ciencia y la tecnología al vivenciar procesos que permiten la exploración y la experimentación.

$\Gamma \quad$ Motivación propia del estudiante durante la elaboración del proyecto.

$\square$ Motivación por parte del docente hacia el estudiante durante la elaboración del proyecto.

$\square$ Visita a algún laboratorio o centro de investigación para realizar el proyecto de feria.

$\Gamma \quad$ Área temática del proyecto.

$\square \quad$ La interacción con el público y con los jueces durante la exposición del proyecto.

$\Gamma$ Obtención de algún reconocimiento en alguna de las etapas de la feria.

$\lceil\quad$ Participación en la Feria Internacional de Ciencia e Ingeniería u otras.

$\ulcorner\quad$ La feria inspira para estudiar carreras científicas y tecnológicas y desempeñarse a futuro en trabajos relacionados con esas áreas.

$\Gamma \quad$ Ninguno de los anteriores.

Otro (especifique)

2. ¿Cuál o cuáles acciones ha desarrollado usted como docente o tutor en el proceso de Ferias de Ciencia y Tecnología para motivar el desarrollo de las vocaciones científicas y tecnológicas en el estudiantado de undécimo y duodécimo nivel?

3. ¿Cuáles aspectos considera usted que deben fortalecerse en el proceso de Ferias de Ciencia y Tecnología para mejorar la promoción de éstas en las vocaciones científicas y tecnológicas y por tanto en la escogencia de carrera universitaria? 